\title{
INVERSION DE LA TRANSFORMATION DE POMPEIU DANS LE DISQUE HYPERBOLIQUE (Cas de deux disques)
}

\author{
Mimoun El Harchaoui
}

\begin{abstract}
In this paper we extend the result established for the euclidean space in [3] to the hyperbolic disk. This includes the reconstruction of a function defined in a fixed disk $B(0, R)$ from its averages on disks of radii $r_{1}, r_{2}$ lying in $B(0, R)$.
\end{abstract}

\section{Introduction}

Nous proposons dans ce travail d'étudier le problème inverse de Pompeiu local des deux disques dans le disque hyperbolique. Il s'agit de reconstituer un signal $f$ connaissant ses moyennes calculées sur les translatées de deux boules géodésiques contenues dans une boule de rayon fixe.

Rappelons le problème de Pompéiu des deux disques dans le cas euclidien. Si $\chi_{1}$ et $\chi_{2}$ désignent les fonctions caractéristiques des boules euclidiennes $B\left(0, r_{1}\right)$ et $B\left(0, r_{2}\right)$, on définit l'application:

$$
\begin{aligned}
P: \mathcal{E}\left(\mathbb{R}^{n}\right) & \longrightarrow \mathcal{E}^{2}\left(\mathbb{R}^{n}\right) \\
f & \longmapsto\left(\chi_{1} * f, \chi_{2} * f\right)
\end{aligned}
$$

appelée traditionnellement transformation de Pompéiu.

Si $P$ est injective, on dit que la famille $\left(B\left(0, r_{1}\right), B\left(0, r_{2}\right)\right)$ possède la propriéte de Pompéiu globale. Si on désigne par $E_{n}$ l'ensemble

$$
E_{n}=\left\{\frac{\xi}{\eta}: \xi>0, \eta>0, J_{\frac{n}{2}}(\xi)=J_{\frac{n}{2}}(\eta)=0\right\}
$$

où $J_{\frac{n}{2}}$ est la fonction Bessel d'indice $\frac{n}{2}$. On a: 
1.1 Théorème ([5], [9]). La famille $\left(B\left(0, r_{1}\right), B\left(0, r_{2}\right)\right)$ possède la propriéte de Pompéiu globale si et seulement si $\frac{r_{2}}{r_{2}} \notin E_{n}$.

Nous allons maintenant préciser ce que nous entendons par la propriété de Pompéiu locale. Soient $B(0, R)$ la boule euclidienne centrée à l'origine de rayon $R$ et $0<r_{1}, r_{2}<R$. On définit de la même façon l'application

$$
\begin{aligned}
P: \mathcal{E}(B(0, R)) & \longrightarrow \mathcal{E}\left(B\left(0, R-r_{1}\right)\right) \times \mathcal{E}\left(B\left(0, R-r_{2}\right)\right) \\
f & \longmapsto\left(\chi_{1} * f, \chi_{2} * f\right)
\end{aligned}
$$

Si $P$ est injective, on dit que $\left(B\left(0, r_{1}\right), B\left(0, r_{2}\right)\right)$ possède la propriété de Pompéiu locale par rapport à $B(0, R)$.

Il est clair que si la propriété globale est non verifiee alors lá propriété locale ne saurait l'être. Le résultat local suivant est connu:

1.2 Théorème ([3], [2]). Soient $r_{1}, r_{2}>0$ tels que $\frac{r_{1}}{r_{2}} \notin E_{n}$ et $R>r_{1}+r_{2}$ alors la famille $\left(B\left(0, r_{1}\right), B\left(0, r_{2}\right)\right)$ possède la propriété de Pompéiu locale par rapport à $B(0, R)$.

Plus précisement, sous les hypotheses du theròme 1.2, il existe un procédé explicite permettant de construire deux suites d'approximations $\left(\nu_{l}\right)_{l \geq 1},\left(\mu_{l}\right)_{l \geq 1}$ à support compact telles que:

$$
\lim _{l \rightarrow+\infty}\left(\nu_{l} *\left(\chi_{1} * f\right)+\mu_{l} *\left(\chi_{2} * f\right)\right)=f
$$

Cela permet, d'après $([3],[2])$, de déterminer $f$ dans $B(0, R)$ à partir de ses moyennes locales $\chi_{1} * f$ et $\chi_{2} * f$.

Dans le cadre des espaces symétriques de type non compact $X=G / K$ ou $G$ est un groupe de Lie semi-simple connexe non compact do centre fini et $K$ un sous groupe compact maximal de $G$, on définit, de façon anałogue, la transformation de Pompéiu:

$$
\begin{aligned}
P: \mathcal{E}(X) & \longrightarrow \mathcal{E}^{2}(X) \\
f & \longmapsto\left(P_{1}(f), P_{2}(f)\right)
\end{aligned}
$$

avec

$$
P_{j}(f)(z)=\int_{g B_{j}} f(x) d x, \quad j=1,2
$$


où $z \in X, g \in G: g .0=z, B_{1}, B_{2}$ deux boules géodésiques centrées en $\pi(e)=0 \in X$ et de rayons respectifs $r_{1}, r_{2}$.

La définition de $P$ peut aussi s'écrire sous la forme d'un système de produit de convolution sur $G$ :

$$
P(f)=\left(\bar{f} * \check{\bar{\chi}}_{B_{1}}, \bar{f} * \overline{\bar{\chi}}_{B_{2}}\right) .
$$

(Consulter la Section 3 pour la définition des opérations * et -).

Dans ce cas le resultat d'injectivité global est bien connu:

1.3 Théorème ([4]). Soit $X$ un espace symétrique de type non compact de rang 1 .

Supposons que $f \in L_{\mathrm{Loc}}^{1}(X)$ et

$$
\int_{B} f(x) d x=0
$$

pour toute boule géodésique dans $X$ de rayon $r_{1}$ ou $r_{2}$, alors $f=0$ si et seulement si les équations $\varphi_{\lambda}^{(\alpha+1, \beta+1)}\left(r_{j}\right)=0, j=1,2$ n'ont aucune solution commune $\lambda \in \mathbb{C}$.

Le but de cet article est de généraliser le résultat établi dans [3] au cas du disque hyperbolique. L'injectivité locale sera alors une conséquence du résultat principal que l'on énonce maintenant en supposant que le signal à restituer est de classe $C^{\infty}$, cas auquel on peut se ramener par regularisation (cf [3, pg 273]).

Théorème. Soient $0<r_{1}<r_{2},\left(r_{1}+r_{2}<R\right)$, tels que les équations

$$
\varphi_{\lambda}^{(1,1)}\left(r_{j}\right)=0, j=1,2
$$

n'aient aucune solution commune $\lambda \in \mathbb{C}$ et $\left(\varepsilon_{p}\right)_{p \geq 1}$ une suite strictement croissante de reels positifs et de limite $\frac{R}{r_{1}+r_{2}}-1, R_{p}=\left(r_{1}+r_{2}\right)(1+$ $\left.\varepsilon_{p}\right), p \geq 1, R_{0}=0$, et telle que

$$
\lim _{p \rightarrow+\infty} \frac{R-R_{p-1}}{R-R_{p}}=1
$$

Pour tout $p \geq 1, r \in\left[R_{p-1}, R_{p} \mid\right.$ et $k \in \mathbb{Z}^{*}$, il existe deux suites de distributions $\left(\mathcal{U}_{l}\right),\left(\mathcal{B}_{l}\right)$ d'ordre $\leq 5$ à support compact dans $B(0, R-$ 
$\left.r_{1}\right), B\left(0, R-r_{2}\right)$ respectivement telles que pour $l \geq \max \left(L_{k}, k\right)$ et $f \in$ $C^{\infty}(B(0, R))$ on ait:

$$
\begin{aligned}
&\left|\frac{1}{2 \pi} \int_{0}^{2 \pi} f\left(\rho e^{i \theta}\right) e^{-i k \theta} d \theta-\left\langle\mathcal{U}_{l}, f \times \check{\mu}_{r_{1}}\right\rangle-\left\langle\mathcal{B}_{l}, f \times \check{\mu}_{r_{2}}\right\rangle\right| \\
& \leq \frac{\gamma}{l} \frac{1}{(R-r)^{7}} \sup _{\substack{z \in B\left(0, R_{p}^{\prime}\right) \\
0 \leq i, j \leq 3}}\left|\frac{\partial^{i+j}}{\partial z^{i} \partial \bar{z}^{j}} f(z)\right|
\end{aligned}
$$

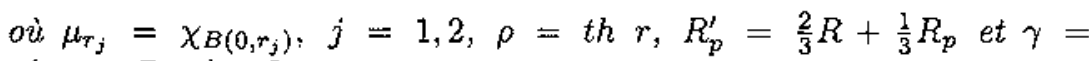
$\gamma\left(r_{1}, r_{2}, R, \varepsilon_{1}\right)>0$.

(Consulter la Section 3 pour la définition de l'opération $\times$ ).

Le cas des espaces symétriques de type non compact de rang $1\left(\mathbb{H}^{n}(\mathbb{R})\right.$, $\left.\mathbb{H}^{n}(\mathbb{C}), \ldots\right)$ sera traité dans un travail ultérieur en préparation.

\section{Notations}

On utilise dans ce travail les notations classiques qu'on rappelle brièvement pour la commodité du lecteur.

Si $G$ est un groupe de Lie on désigne par:

$\mathcal{E}(G)$ : L'espace des fonctions $C^{\infty}$ sur $G$.

$\mathcal{D}(G)$ : L'espace des fonctions $C^{\infty}$ sur $G$ à support compact.

$\mathcal{E}(D)$ : L'espace des fonctions $C^{\infty}$ sur $D$.

$\mathcal{D}(D)$ : L'espace des fonctions $C^{\infty}$ sur $D$ à support compact.

$\mathcal{E}^{\prime}(D)$ : L'espace des distributions à support compact dans $D$.

$\mathcal{E}_{0}^{\prime}(D)$ : L'espace des distributions radiales à support compact dans $D$.

$\mathcal{H}(\mathbb{C})$ : L'espace des fonctions entières.

$B(0, r)$ : La boule géodésique centrée à l'origine et de rayon $r$.

$B_{e}(0, r)$ : La boule euclidienne centrée à l'origine et de rayon $r$.

Si $T \in \mathcal{D}^{\prime}(G)$ et $f \in \mathcal{D}(G)$ on désigne par $\check{T}$ la distribution: $\langle\check{T}, f\rangle=$ $\langle T, \dot{f}\rangle$ avec $\tilde{f}(g)=f\left(g^{-1}\right), \quad g \in G$.

\section{Rappels sur l'analyse harmonique dans le disque hyperbolique}

On appelle disque hyperbolique, le disque unité ouvert de $\mathbb{C}, D=\{z \in$ $\mathbb{C}:|z|<1\}$, muni de la structure riemannienne définie par le produit scalaire \langle\rangle$_{z}$ sur l'espace tangent $T_{z}(D)=\mathbb{R}^{2}$ à $D$ en $z$

$$
\langle u, v\rangle_{2}=\frac{(u \mid v)}{\left(1-|z|^{2}\right)^{2}}
$$


ou $(u \mid v)$ est le produit scalaire euclidien usuel. Si on désigne par $d$ la distance riemannienne définie par ce procédé on $a$, pour tout $z_{1}, z_{2} \in D$

$$
d\left(z_{1}, z_{2}\right)=d\left(0, \frac{\left|z_{2}-z_{1}\right|}{\left|1-\bar{z}_{1} z_{2}\right|}\right), \text { avec } d(0, z)=\frac{1}{2} \log \frac{1+|z|}{1-|z|} .
$$

L'élément de volume est $d \mu(z)=\frac{d m(z)}{\left(1-|z|^{2}\right)^{2}}$ avec $d m(z)=d x d y$ si $z=x+i y$ et le Laplacien-Beltrami est l'opérateur $L=\left(1-|z|^{2}\right)^{2} \Delta$, avec $\Delta=\frac{\partial^{2}}{\partial x^{2}}+\frac{\partial^{2}}{\partial y^{2}}$.

Le disque hyperbolique apparait naturellement comme un espace symétrique de rang 1 obtenu comme quotient de $G=S U(1,1)$ par $S O(2)$ : Le groupe

$$
G=\left\{\left(\begin{array}{ll}
a & b \\
\bar{b} & \bar{a}
\end{array}\right) \in M(2, \mathbb{C}):|a|^{2}-|b|^{2}=1\right\}
$$

opère transitivement sur $D$ par l'opération:

$$
(g, z) \in G \times D \longmapsto g . z=(a z+b)(\bar{b} z+\bar{\alpha})^{-1}
$$

et le stabilisateur de zéro est:

$$
S_{0}=\left\{\left(\begin{array}{cc}
a & 0 \\
0 & \bar{a}
\end{array}\right):|a|^{2}=1\right\} \simeq S O(2)
$$

Il est bien connu [7] que la fonction $e_{\mu, b}(z)$ définie par:

$$
e_{\mu, b}(z)=\left(\frac{1-|z|^{2}}{|z-b|^{2}}\right)^{\frac{\mu}{2}}, \quad|z|<1
$$

avec $\mu \in \mathbb{C}$ et $b \in B=\partial D$, est une fonction propre de $L$ pour la valeur propre $\mu(\mu-2)$.

Si $f: D \longrightarrow \mathbb{C}$ est une fonction mesurable telle que, pour tout $\lambda \in$ $\mathbb{C}, b \in B$ la fonction

$$
z \in D \longmapsto f(z) e_{-i \lambda+1, b}(z)
$$

soit intégrable pour la mesure $d \mu(z)$, on introduit la fonction:

$\tilde{f}: \mathbb{C} \times B \longrightarrow \mathbb{C}$ définie par:

$$
\widetilde{f}(\lambda, b)=\int_{D} f(z) e_{-i \lambda+1, b}(z) d \mu(z)
$$


que l'on appelle transformée de Fourier de $f$. Avec des majorations usuelles on peut obtenir l'holomorphie en $\lambda$.

On dit qu'une fonction $\mathcal{C}^{\infty}, \psi: \mathbb{C} \times B \longrightarrow \mathbb{C}$, holomorphe en $\lambda \in \mathbb{C}$ pour chaque $b \in B$, est de type exponentiel uniforme $R$ si, quel que soit $N \in \mathbb{N}$, on a:

$$
\sup _{(\lambda, b) \in \mathcal{C} \times B} e^{-R|I m \lambda|}(1+|\lambda|)^{N}|\psi(\lambda, b)|<\infty .
$$

On dispose alors de la "version hyperbolique" suivante des résultats usuels concernant l'inversion de la transformation de Fourier et les théorèmes du type "Paley-Wiener".

\subsection{Théorème ([7]).}

i) Si $f \in \mathcal{D}(D)$ on a la formule d'inversion:

$$
f(z)=\frac{1}{4 \pi} \int_{\mathbb{R}} \int_{B} \tilde{f}(\lambda, b) e_{i \lambda+1 ; b}(z) \lambda \operatorname{th}\left(\frac{\pi \lambda}{2}\right) d \lambda d b
$$

où db est lo mesure $\geq 0$, invariante par rotation, de masse 1 sur $B$.

ii) L'application $f \longmapsto \tilde{f}$ est une bijection de $\mathcal{D}(D)$ sur l'espace des fonctions $\psi(\lambda, b)$ de type exponentiel uniforme satisfaisant l'équation fonctionnelle:

$$
\int_{B} e_{i \lambda+1, b}(z) \psi(\lambda, b) d b=\int_{B} e_{-i \lambda+1, b}(z) \psi(-\lambda, b) d b
$$

iii) L'application $f \longmapsto \tilde{f}$ se prolonge en une isométrie de $L^{2}(D, d \mu)$ sur $L^{2}\left(\mathbb{R}^{+} \times B,(2 \pi)^{-1} \lambda t h\left(\frac{\pi \lambda}{2}\right) d \lambda d b\right)$.

Nous allons introduire à présent les concepts de fonctions sphériques sur $D$ et de transformée sphérique.

On dit que $f: D \longrightarrow \mathbb{C}$ est radiale si $f(z)=f(b z)$ pour tout $z \in D$ et $b \in B$. Une fonction sphérique est alors, par définition, unc fonction radiale qui est aussi une fonction propre du Laplacien hyperbolique. On trouvera dans [7], la preuve du fait important suivant:

Toute fonction sphérique est un multiple par un scalaire complexe de la fonction:

$$
\varphi_{\lambda}(z)=\int_{B} e_{i \lambda+1, b}(z) d b
$$

où $\lambda$ est un paramètre complexe. On vérifie que:

$$
L\left(\varphi_{\lambda}\right)(z)=-\left(1+\lambda^{2}\right) \varphi_{\lambda}(z)
$$


On appellera transformée spherique d'une fonction radiale $f$ telle que, pour tout $\lambda \in \mathbb{C} z \longmapsto f(z) \varphi_{-\lambda}(z)$ soit dans $L^{1}(D, d \mu)$, la fonction:

$$
\widetilde{f}(\lambda)=\int_{D} f(z) \varphi_{-\lambda}(z) d \mu(z)
$$

On a alors l'énoncé suivant:

\subsection{Théorème ([7]).}

i) Pour toute fonction radiale $f \in \mathcal{D}(D)$ on a la formule d'inversion:

$$
f(z)=\frac{1}{2 \pi^{2}} \int_{\mathbb{R}} \tilde{f}(\lambda) \varphi_{\lambda}(z)|c(\lambda)|^{-2} d \lambda, \quad z \in D
$$

avec

$$
|c(\lambda)|^{-2}=\frac{\pi \lambda}{2} \operatorname{th}\left(\frac{\pi \lambda}{2}\right) .
$$

ii) On a la formule de Plancherel, pour $f$ telle que ci-dessus:

$$
\int_{D}|f(z)|^{2} d \mu(z)=\frac{1}{2 \pi^{2}} \int_{\mathbb{R}}|\tilde{f}(\lambda)|^{2}|c(\lambda)|^{-2} d \lambda .
$$

iii) L'image par la transformation sphérique des fonctions radiales de $\mathcal{D}(D)$ est l'espace des fonctions entières paires $F$ du type de Paley-Wiener, ie; il existe $R \geq 0$ tel que, pour tout $N \in \mathbb{N}$, on ait:

$$
\sup _{\lambda \in \mathbb{C}} e^{-R|I m \lambda|}(1+|\lambda|)^{N}|F(\lambda)|<\infty
$$

iv) L'application $f \longmapsto \tilde{f}$ se prolonge en une isometrie du sous-espace de $L^{2}(D, d \mu)$ constitué des fonctions radioles, sur le sous espace des fonctions paires de $L^{2}\left(\mathbb{R}^{+}, \frac{1}{2 \pi} \lambda \operatorname{th}\left(\frac{\pi \lambda}{2}\right) d \lambda\right)$.

Nous introduisons maintenant la notion de convolution sur $G=$ $S U(1,1)$ dont nous noterons $d g$ la mesure de Haar pour laquelle on peut remarquer que

$$
\int_{D} f d \mu=\int_{G}(f \circ \pi) d g
$$

pour toute fonction $f \in \mathcal{D}(D)$ où $\pi: G \longrightarrow D$ est la projection canonique. 
Si $f, \varphi \in \mathcal{D}(G)$ on pose

$$
(f * \varphi)(g)=\int_{G} \varphi(h) f\left(g h^{-1}\right) d h=\int_{G} f(h) \varphi\left(h^{-1} g\right) d h .
$$

Comme usuellement, cette convolution s'étend aux distributions. En introduisant la distribution $\delta_{K}$ sur $G$ définie par:

$$
\delta_{K}: f \longmapsto \int_{K} f(k) d k
$$

où $K=S O(2)$ et $d k$ est la mesure de Haar normalisée sur $S O(2)$, on peut associer, à chaque $f \in \mathcal{E}(G)$, une fonction $f_{\pi} \in \mathcal{E}(D)$ définie par:

$$
f_{\pi} \circ \pi=f * \delta_{K}
$$

Chaque $T \in \mathcal{D}^{\prime}(D)$ induit une distribution $\bar{T}$ sur $G$ par la règle:

$$
\langle\bar{T}, f\rangle=\left\langle T, f_{\pi}\right\rangle
$$

(si $x=\pi(g)$ on a $f_{\pi}(x)=\left\langle\left(\delta_{K}\right)_{h}, f\left(g h^{-1}\right)\right\rangle=\int_{K} f\left(g k^{-1}\right) d k=$ $f_{K} f(g k) d k$, et ainsi le support de $f_{\pi}$ est contenu dans $\pi(\operatorname{supp}(f))$. En particulier $f_{\pi} \in \mathcal{D}(D)$ si $f \in \mathcal{D}(G)$. Si une fonction est considérée comme une distribution on a: $\bar{\varphi}(g)=\varphi(\pi(g))$, soit $(\bar{\varphi})_{\pi}=\varphi$.

Ainsi, les fonctions, (ou les distributions) sur $X$ peuvent être identifiees avec les fonctions, (ou les distributions) sur $G$ qui sont invariantes à droite sous l'action de $K$ (ie: $\varphi(g k)=\varphi(g)$ pour tout $k \in K$ ). On a aussi une notion de convolution dans $\mathcal{D}^{\prime}(D)$ donnée par:

$$
T_{1} \times T_{2}=\bar{T}_{1} * \bar{T}_{2}
$$

si l'une des distributions $T_{j}$ a son support compact. Cette convolution est associative et vérifie $T \times \delta=\delta \times T=T$ où $\delta$ est la mesure de Dirac: à l'origine.

On dit qu'une fonction $f$ sur $G$ est $K$-bi-invariante si $f\left(k_{1} g k_{2}\right)=f(g)$ pour tout $g \in G$ et $k_{1}, k_{2} \in K$. Si $f \in \mathcal{D}(G)$ est $K$-bi-invariante, on dispose d'une "Transformée Sphérique" $\mathcal{F}$ donnée par:

$$
\mathcal{F}(f)(\lambda)=\int_{G} f(g) \varphi_{\lambda}\left(g^{-1}\right) d g
$$

où $\varphi_{\lambda}(g)=\varphi_{\lambda}(\pi(g))$ par abus de langage. Il est clair que $f_{\pi}$ est radiale et élément de $\mathcal{D}(D)$ et que $\widetilde{f_{\pi}}=\mathcal{F}(f)$. 
Nous noterons $\mathcal{D}_{0}(G)$ l'ensemble des éléments $K$-bi-invariants de $\mathcal{D}(G)$.

On a un analogue du théorème de Paley-Wiener:

La transformation de Fourier sphérique établit une bijection de $\mathcal{D}_{0}(G)$ sur l'espace des fonctions entières paires de type de Paley-Wiener.

Cette bijection se prolonge en un isomorphisme linéaire entre l'espace $\mathcal{E}_{0}^{\prime}(G)$ des distributions $K$-bi-invariantes à support compact dans $G$ et l'espace des fonctions entières paires à croissance polynomiale sur $\mathbb{R}$. La correspondance entre $\mathcal{E}_{0}^{\prime}(G)$ et $\mathcal{F}\left(\mathcal{E}_{0}^{\prime}(G)\right)$ est un isomorphisme topologique d'algèbres ([7]).

Nous aurons l'occasion d'utiliser les fonctions $\varphi_{\lambda}^{(\alpha, \beta)}(r)$ définies par:

$$
\varphi_{\lambda}^{(\alpha, \beta)}(r)=F\left(\frac{1}{2}(\rho+i \lambda), \frac{1}{2}(\rho-i \lambda) ; \alpha+1 ;-(s h r)^{2}\right)
$$

où $F$ est la fonction hypergéométrique de première espèce.

Rappelons que la fonction $F$ admet la représentation intégrale.

$$
F(\alpha, \beta ; \gamma ; z)=\frac{\Gamma(\alpha)}{\Gamma(\beta) \Gamma(\gamma-\beta)} \int_{0}^{1} t^{\beta-1}(1-t)^{\gamma-\beta-1}(1-t z)^{-\alpha} d t
$$

pour $\operatorname{Re} \gamma>\operatorname{Re} \beta>0$.

Ici $\rho=\alpha+\beta+1$, et la fonction $\varphi_{\lambda}$ n'est autre que $\varphi_{\lambda}^{(0,0)}$ (cf: [7]). (on renvoie à $[6]$ pour les fonctions hypegéométriques).

Nous utilisons de façon fréquente dans la suite le résultat du cálcul suivant que nous rappelons pour la commodité du lecteur:

3.3 Lemme $([4])$. Soit $\chi_{B(0, r)}$ l'indicatrice de la boule géodésique centrée à l'origine et de rayon $r>0$. La distribution $\chi_{B(0, r)}$ est radiale et a pour transformée sphérique la fonction

$$
\widetilde{\chi}_{B(0, r)}(\lambda)=\pi(s h r)^{2}(c h r)^{2} \varphi_{\lambda}^{(1,1)}(r) .
$$

Preuve: On a

$$
\tilde{\chi}_{B(0, r)}(\lambda)=\int_{B(0, r)} \varphi_{\lambda}(z) d \mu(z)
$$

en coordonnées géodésiques polaires, $t=d(0, z)$ et $\theta$, on a $z=($ th $t) e^{i \theta}$ avec $|z|=$ th $t$. La mesure $d \mu$ s'ecrit ([7, page 84])

$$
d \mu(z)=\frac{1}{2} \operatorname{sh} 2 t d t d \theta
$$


Ainsi

$$
\begin{aligned}
\tilde{\chi}_{B(0, r)}(\lambda)=\frac{1}{2} \int_{0}^{r} \int_{0}^{2 \pi} \varphi_{\lambda}(t h t) & \operatorname{sh} 2 t d t d \theta \\
& =\pi \int_{o}^{r} F\left(\nu, 1-\nu ; 1 ;-(\operatorname{sh} t)^{2}\right) \operatorname{sh} 2 t d t
\end{aligned}
$$

avec $\nu=\frac{1}{2}(1+i \lambda)$. D'après $([\mathbf{6},(22)$ page 102]) on a

$$
F(\nu, 1-\nu ; 1 ; z)=\frac{d}{d z}[z F(\nu, 1-\nu ; 2 ; z)]
$$

et il vient

$$
\operatorname{sh} 2 t F\left(\nu, 1-\nu ; 1 ;-(s h t)^{2}\right)=\frac{d}{d t}\left[(s h t)^{2} F\left(\nu, 1-\nu ; 2 ;-(s h t)^{2}\right)\right]
$$

d'où

$$
\tilde{\chi}_{B(0, r)}(\lambda)=\pi(s h r)^{2} F\left(\nu, 1-\nu ; 2 ;-(s h r)^{2}\right) .
$$

Enfin, en utilisant l'égalité [6].

$$
F(a, b ; c ; z)=(1-z)^{c-a-b} F(c-a, c-b ; c ; z),
$$

on obtient

$$
\pi(s h r)^{2} F\left(\nu, 1-\nu ; 2 ;-(s h r)^{2}\right)=\pi(s h r)^{2}(c h r)^{2} \varphi_{\lambda}^{(1,1)}(r) .
$$

3.4 Lemme ([4]). La transformée sphérique de la distribution radiale $\sigma_{r}$, mesure d'intégration normalisée sur $\partial B(0, r)$ pour l'elément de longueur hyperbolique, est:

$$
\widetilde{\sigma}_{r}(\lambda)=\varphi_{\lambda}^{(0,0)}(r)
$$

Preuve: On a

$$
\tilde{\sigma}_{r}(\lambda)=\int_{\partial B(0, r)} \varphi_{\lambda}(s) d \sigma_{r}(s)=\varphi_{\lambda}^{(0,0)}(r) \int_{\partial B(0, r)} d \sigma_{r}(s)=\varphi_{\lambda}^{(0,0)}(r)
$$




\section{Quelques lemmes preliminaires}

4.1 Lemme. Soient $\left(\left(\frac{\partial}{\partial \bar{z}}\right)^{n}\right)^{*}$, (respectivement $\left(\left(\frac{\partial}{\partial z}\right)^{n}\right)^{*}$, l'operateur differentiel ädjoint de $\left(\frac{\partial}{\partial \bar{z}}\right)^{n},\left(\right.$ respectivement de $\left.\left(\frac{\partial}{\partial z}\right)^{n}\right)$, conçu comme opérateur differentiel hyperbolique, et $\delta_{0}$ la distribution dans $D$ définie par: $\delta_{0}(f)=f(0), f \in \mathcal{E}(D)$. On a

$$
\begin{aligned}
& \left(\left(\frac{\partial}{\partial \bar{z}}\right)^{n}\right)^{*} \delta_{0}=(-1)^{n}\left(\frac{\partial}{\partial \bar{z}}\right)^{n} \delta_{0} \\
& \left(\left(\frac{\partial}{\partial z}\right)^{n}\right)^{*} \delta_{0}=(-1)^{n}\left(\frac{\partial}{\partial z}\right)^{n} \delta_{0}
\end{aligned}
$$

Preuve: On désigne par \langle\rangle , (resp \langle\rangle$_{e}$ ), la dualité hyperbolique, (resp. euclidienne).

(i) Soit $f \in \mathcal{D}(D)$ on a:

$$
\begin{aligned}
\left\langle\left(\left(\frac{\partial}{\partial \bar{z}}\right)^{n}\right)^{*} \delta_{0}, f\right\rangle & =\left\langle\delta_{0},\left(\frac{\partial}{\partial \bar{z}}\right)^{n} f\right\rangle \\
& =\left\langle\delta_{0}, \frac{1}{\left(1-|z|^{2}\right)^{2}}\left(\frac{\partial}{\partial \bar{z}}\right)^{n} f\right\rangle_{e} \\
& =\left\langle\delta_{0},\left(\frac{\partial}{\partial \bar{z}}\right)^{n} f\right\rangle_{e} \\
& =\left\langle(-1)^{n}\left(\frac{\partial}{\partial \bar{z}}\right)^{n} \delta_{0}, f\right\rangle_{e} \\
& =\left\langle(-1)^{n}\left(\frac{\partial}{\partial \bar{z}}\right)^{n} \delta_{0}, \frac{1}{\left(1-|z|^{2}\right)^{2}} f\right\rangle_{e} \\
& =\left\langle(-1)^{n}\left(\frac{\partial}{\partial \bar{z}}\right)^{n} \delta_{0}, f\right\rangle
\end{aligned}
$$

ce qui prouve (i). La preuve du point (ii) résulte de (i) en remplaçant $\bar{z}$ par $z$.

4.2 Lemme. Soit $S_{n}$ la distribution définie par:

$$
S_{n}= \begin{cases}\left(\left(\frac{\partial}{\partial z}\right)^{n}\right)^{*} \delta_{0}, & \text { si } n \geq 0 \\ \left(\left(\frac{\partial}{\partial \bar{z}}\right)^{|n|}\right)^{*} \delta_{0}, & \text { si } n \leq 0\end{cases}
$$

on $a$ :
(i) $\widetilde{S}_{n}(\lambda, b)=\bar{b}^{n} P_{|n|}(-i \lambda)$ 
(ii) $\check{S}_{n}=(-1)^{n} S_{n}$

où: $P_{n}(-i \lambda)=(1-\nu)((1-\nu)+1)((1-\nu)+2) \ldots((1-\nu)+n-1)=$ $\frac{\Gamma((1-\nu)+n)}{\Gamma(1-\nu)}$, avec $\nu=\frac{1}{2}(1+i \lambda)$ et $P_{n}(x)=\left(\frac{1}{2}(x+1)\right)\left(\frac{1}{2}(x+1)+\right.$ 1) $\ldots\left(\frac{1}{2}(x+1)+n-1\right)$.

Preuve: (i) si $n \geq 0$ on a:

$$
\begin{aligned}
\widetilde{S}_{n}(\lambda, b) & =\left\langle\left(\left(\frac{\partial}{\partial z}\right)^{n}\right)^{*} \delta_{0}, e_{1-i \lambda, b}(z)\right\rangle \\
& =\left\langle\delta_{0},\left(\frac{\partial}{\partial z}\right)^{n} e_{1-i \lambda, b}(z)\right\rangle \\
& =\left(\frac{\partial}{\partial z}\right)^{n}\left[e_{1-i \lambda, b}(z)\right]_{z=0} \\
& =\left(\frac{\partial}{\partial z}\right)^{n}\left[\left(\frac{1-|z|^{2}}{|z-b|^{2}}\right)^{\frac{1}{2}(1-i \lambda)}\right]_{z=0} \\
& =\bar{b}^{n}(1-\nu)((1-\nu)+1)((1-\nu)+2) \ldots((1-\nu)+n-1) \\
& =\bar{b}^{n} P_{n}(-i \lambda) .
\end{aligned}
$$

Dans le cas $n \leq 0$ on montre, de la même façon, que

$$
\tilde{S}_{n}(\lambda, b)=b^{|n|} P_{|n|}(-i \lambda)=\bar{b}^{n} P_{|n|}(-i \lambda) .
$$

Pour (ii) on a

$$
\begin{aligned}
\widetilde{\widetilde{S}}_{n}(\lambda, b) & =\left\langle\breve{S}_{n}, e_{1-i \lambda}(z)\right\rangle \\
& =\left\langle S_{n}, e_{1-i \lambda}(-z)\right\rangle \\
& =(-\bar{b})^{n} P_{n}(-i \lambda)=(-1)^{n} \widetilde{S}_{n}(\lambda, b) .
\end{aligned}
$$

4.3 Lemme. Soit $H_{k}$ le polynôme défini par

$$
H_{k}(w)= \begin{cases}\bar{w}^{k} & \text { si } k>0 \\ w^{|k|} & \text { si } k<0\end{cases}
$$

et $\sigma_{r}$ la mesure d'intégration de masse totale égale à 1 sur $\partial B(0, r)$, bord de la boule géodésique centrée à l'origine et de rayon $r>0$ dans $D\left(d \sigma_{r}=\frac{d \theta}{2 \pi}\right)$ considérée comme distribution radiale dans $D$ (pour la structure hyperbolique). On a

$$
H_{k} \sigma_{r}=\frac{1}{\pi(|k|-1) !(c h r)^{2}} S_{k} \times T_{\rho, k}
$$


où $T_{\rho, k}$ est la distribution radiale dans $D$ défnie par:

$$
T_{\rho, k}(w)=\left(\frac{\rho^{2}-|w|^{2}}{1-|w|^{2}}\right)^{|k|-1} \chi_{B(0, r)}(w) \quad(|k| \geq 1)
$$

où $w \in D$ et $\rho=$ th $r$ et $S_{k}$ est définie dans le lemme 4.2.

Preuve: Nous remarquons que si $w=\rho e^{i \theta}$, on a $H_{k}(w)=\rho^{|k|} e^{-i k \theta}$ pour tout $k \in \mathbb{Z}^{*}$

$$
\begin{aligned}
\widehat{H_{k} \sigma_{r}}(\lambda, b) & =\left\langle H_{k} \sigma_{r}, e_{-i \lambda+1, b}\right\rangle \\
& =\int_{\partial B(0, r)} H_{k}(w) e_{-i \lambda+1_{1} b}(w) d \sigma_{r}(w) \\
& =\frac{\rho^{|k|}}{2 \pi} \int_{0}^{2 \pi} e_{-i \lambda+1, b}\left(\rho e^{i \theta}\right) e^{-i k \theta} d \theta, \text { avec } \rho=t h r \\
& =\frac{\rho^{|k|}}{2 \pi} \int_{0}^{2 \pi}\left(\frac{1-\rho^{2}}{\left|\rho e^{i \theta}-b\right|^{2}}\right)^{1-\nu} e^{-i k \theta} d \theta \\
& =\bar{b}^{k} \frac{\rho^{|k|}}{2 \pi} \int_{0}^{2 \pi}\left(\frac{1-\rho^{2}}{\left|\rho-e^{i \theta}\right|^{2}}\right)^{1-\nu} e^{i k \theta} d \theta,
\end{aligned}
$$

or, d'après $([7])$, on a pour tout $k \in \mathbb{Z}$,

$$
\begin{array}{r}
\frac{1}{2 \pi} \int_{0}^{2 \pi}\left(\frac{1-\rho^{2}}{\left|\rho-e^{i \theta}\right|^{2}}\right)^{1-\nu} e^{i k \theta} d \theta \\
=\frac{\rho^{|k|} \Gamma(|k|+1-\nu)}{|k| ! \Gamma(1-\nu)} F\left(\nu, 1-\nu ;|k|+1 ;-(s h r)^{2}\right)
\end{array}
$$

Par suite

$$
\widetilde{H_{k} \sigma_{r}(\lambda, b)}=\frac{\bar{b}^{k} \rho^{2|k|}}{|k| !} P_{|k|}(-i \lambda) F\left(\nu, 1-\nu ;|k|+1 ;-(s h r)^{2}\right) .
$$

D'autre part, d'après le lemme 4.2 , on a

$$
{\widetilde{S_{k} \times T_{\rho, k}}}_{(\lambda, b)} \widetilde{S}_{k}(\lambda, b), \tilde{T}_{\rho, k}(\lambda)=\bar{b}^{k} P_{[k\}}(-i \lambda) \widetilde{T}_{\rho, k}(\lambda) .
$$

Pour finir la preuve, nous allons calculer la transformée sphérique de 
$T_{p, k}$. On a

$$
\begin{aligned}
\tilde{T}_{\rho, k}(\lambda) & =\int_{B(0, r)}\left(\frac{\rho^{2}-|w|^{2}}{1-|w|^{2}}\right)^{|k|-1} \varphi_{-\lambda}(w) d \mu(w) \\
& =\int_{B(0, \rho)}\left(\frac{\rho^{2}-|w|^{2}}{1-|w|^{2}}\right)^{|k|-1}\left(1-|w|^{2}\right)^{\nu-2} F\left(\nu, \nu ; 1 ;|w|^{2}\right) d m(w) \\
& =2 \pi \int_{0}^{\rho}\left(\rho^{2}-s^{2}\right)^{|k|-1}\left(1-s^{2}\right)^{\nu-1-|k|} F\left(\nu, \nu ; 1 ; s^{2}\right) s d s \\
& =\pi \int_{0}^{\rho^{2}}\left(\rho^{2}-s\right)^{|k|-1}(1-s)^{\nu-1-|k|} F(\nu, \nu ; I ; s) d s .
\end{aligned}
$$

D'après ([1, Théorème 2.3 , page 418]) on a: si $\mu>0, c>0$, et $0<y<1$ alors:

$$
\begin{aligned}
y^{c+\mu-1}(1-y)^{a-c} F(a, b+\mu ; c+\mu ; y) \\
=\frac{\Gamma(c+\mu)}{\Gamma(c) \Gamma(\mu)} \int_{0}^{y}(y-x)^{\mu-1}(1-x)^{a-c-\mu} x^{c-1} F(a, b ; c ; x) d x .
\end{aligned}
$$

On applique cette relation avec $y=\rho^{2}, \mu=|k|, c=1$ et $a=b=\nu$. On trouve

$$
\begin{aligned}
\widetilde{T}_{\rho, k}(\lambda) & =\frac{\pi}{|k|} \rho^{2|k|}\left(1-\rho^{2}\right)^{\nu-1} F\left(\nu, \nu+|k| ;|k|+1 ; \rho^{2}\right) \\
& =\frac{\pi}{|k|} \rho^{2|k|}(c h r)^{2} F\left(\nu, 1-\nu ;|k|+1 ;-(s h r)^{2}\right)
\end{aligned}
$$

la derniere égalité résulte de la rclation: [6, page 64 (22)]

$$
F(a, b ; c ; x)=(1-x)^{-a} F\left(a, c-b ; c ; \frac{x}{x-1}\right) \text { pour }|x|<1 .
$$

Finalement, d'après (23), (25) et le lemme 4.2, on a:

$$
\widehat{H_{k} \sigma_{r}}(\lambda, b)=\frac{1}{\pi(|k|-1) !(c h r)^{2}} \widetilde{S}_{k}(\lambda, b), \widetilde{T}_{\rho ;}(\lambda)
$$

d'où, compte-tenu des lemmes 4.1 et 4.2 :

$$
\begin{aligned}
H_{k} \sigma_{r}=\frac{1}{\pi(|k|-1) !(c h r)^{2}} S_{k} \times T_{\rho, k} \\
\quad= \begin{cases}\frac{(-1)^{k}}{\pi(k-1) !(c h r)^{2}}\left(\left(\frac{\partial}{\partial z}\right)^{k} \delta_{0}\right) \times T_{\rho, k} & \text { si } k>0 \\
\frac{(-1)^{k}}{\pi(|k|-1) !(c h r)^{2}}\left(\left(\frac{\partial}{\partial z}\right)^{|k|} \delta_{0}\right) \times T_{\rho, k} & \text { si } k<0\end{cases}
\end{aligned}
$$

d'où le lemme. 
4.4 Lemme. Soient $a_{1}, a_{2}, a_{3}$ trois nombres réels strictement positifs, $k$ un entier positif ou nul et

$$
\theta(\lambda)=\varphi_{\lambda}^{(1,1)}\left(a_{1}\right) \varphi_{\lambda}^{(1,1)}\left(a_{2}\right) \varphi_{\lambda}^{(k, k)}\left(a_{3}\right)
$$

Il existe des constantes $L_{k}, A_{k}>0$ telles que, pour tout entier $l \geq L_{k}$, il existe $\left.\rho_{l} \in\right] l, l+1[$ tel que:

(i) $S i|\operatorname{Im} \lambda| \geq 1$ et $|\lambda| \geq L_{k}$ ou bien

(ii) $S i|\lambda|=\rho_{l}$ on ait:

$$
|\theta(\lambda)| \geq \frac{A_{k}}{|\lambda|^{k+\frac{7}{2}}} e^{\left(a_{1}+a_{2}+a_{3}\right)|\operatorname{Im} \lambda|}
$$

De plus, si $\delta>0$ est donné et si $a_{1}, a_{2}, a_{3} \in\left[\delta, \delta^{-1}\right]$, on peut choisir $A_{k}$ et $L_{k}$ dépendant seulement de $\delta$ et de $k$.

Enfin on peut choisir la suite $\left(\rho_{l}\right)_{l \geq L_{k}}$ dépendant seulement de $a_{1}, a_{2}$, $a_{3}$ et de $k$.

Preuve: Puisque la fonction $\theta$ est paire il suffit d'effectuer la preuve pour $\operatorname{Re} \lambda \geq 0$.

Nous allons utiliser le développement asymptotique de $F(\nu, 1-\nu ; k+$ $\left.1,-(s h t)^{2}\right)$ par rapport à la variable $\nu[8]$ :

Pour $z=c h \zeta, \zeta=\xi+i \eta$ où $\xi \geq 0$ et $|\eta| \leq \pi$ on a:

$$
\begin{aligned}
& \text { (30) } F\left(\alpha+\tau, \beta-\tau ; \gamma ; \frac{1}{2}(1-z)\right) \\
& \sim \frac{\Gamma(1-\beta+\tau) \Gamma(\gamma)}{\pi \Gamma(\gamma-\beta+\tau)} 2^{\alpha+\beta-1}\left(1-e^{-\zeta}\right)^{\frac{1}{2}-\gamma}\left(1+e^{-\zeta}\right)^{\gamma-\alpha-\beta-\frac{1}{2}} \\
& \times\left[e^{(\tau-\beta) \zeta} \sum_{s=0}^{\infty} c_{s} \Gamma\left(s+\frac{1}{2}\right) \tau^{-s-\frac{1}{2}}+e^{ \pm i \pi\left(\gamma-\frac{1}{2}\right)} e^{-(\tau+\alpha) \zeta} \sum_{s=0}^{\infty} c_{s}^{\prime} \Gamma\left(s+\frac{1}{2}\right) \tau^{-s-\frac{1}{2}}\right]
\end{aligned}
$$

valable pour $|\tau|$ assez grand et $-\frac{\pi}{2}-\omega_{2}+\delta<\operatorname{Arg} \tau<\frac{\pi}{2}+\omega_{1}-\delta$, pour tout $\delta>0$ et ou

$$
\left\{\begin{array}{lll}
\omega_{2}=\operatorname{Arctg}\left(\frac{\eta}{\xi}\right), & \omega_{1}=-\operatorname{Arctg}\left(\frac{\eta-\pi}{\xi}\right), & \text { si } \eta \geq 0 \\
\omega_{2}=\operatorname{Arctg}\left(\frac{\eta+\pi}{\xi}\right), & \omega_{1}=-\operatorname{Arctg}\left(\frac{\eta}{\xi}\right), & \text { si } \eta \leq 0 .
\end{array}\right.
$$

On désigne ici par Arctg la détermination principale de Arctg : $-\frac{\pi}{2}<$ $\operatorname{Arctg}<\frac{\pi}{2}$. 
Dans le cas particulier où $\alpha=\beta=\frac{1}{2}, \gamma=k+1, z=\operatorname{ch} 2 t(t \geq 0)$ et $r=\frac{i \lambda}{2}$ on $a: c_{0}=c_{0}^{\prime}=1,1-\nu=\frac{1}{2}(1-i \lambda)=\beta-\tau$ et $\nu=\alpha+\tau=$ $\frac{1}{2}(1+i \lambda)$. Par suite (30) s'écrit:

$$
\begin{aligned}
& F\left(\nu, 1-\nu ; k+1 ;-(s h t)^{2}\right) \\
= & C(\lambda, k, t)\left[\left(e^{i\left(\lambda t-\frac{\pi}{4}\right)}+e^{ \pm\left(k+\frac{1}{2}\right) i \pi} e^{-i\left(\lambda t+\frac{\pi}{4}\right)}\right) \frac{1}{\lambda^{\frac{1}{2}}}+O\left(\frac{e^{|I m \lambda| t}}{|\lambda|^{\frac{3}{2}}}\right)\right]
\end{aligned}
$$

avec

$$
C(\lambda, k, t)=\frac{\Gamma(\nu) k !}{\Gamma(\nu+k) \sqrt{\pi}}(t h t)^{-k}(\operatorname{sh} 2 t)^{-\frac{1}{2}},
$$

soit encore, en ne conservant que le signe + dans les exponentielles $e^{ \pm\left(k+\frac{1}{2}\right) i \pi}$, ce qui est possible car $\operatorname{Im}(\operatorname{ch} 2 t)=0([\mathbf{8}$, page 289$])$ :

$$
\begin{aligned}
& F\left(\nu, 1-\nu ; k+1 ;-(s h t)^{2}\right) \\
& \quad=C(\lambda, k, t)\left[\frac{2(i)^{k}}{\lambda^{\frac{1}{2}}} \cos \left(\lambda t-(2 k+1) \frac{\pi}{4}\right)+O\left(\frac{e^{|1 m \lambda| t}}{|\lambda|^{\frac{3}{2}}}\right)\right] .
\end{aligned}
$$

Ces développements sont valables pour:

$$
\left\{\begin{array}{l}
-\pi+\delta<\operatorname{Arg} \lambda<\delta+\operatorname{Arctg}\left(\frac{\pi}{2 r}\right) \\
\quad \text { ou bien } \\
-\pi+\delta-\operatorname{Arctg}\left(\frac{\pi}{2 r}\right)<\operatorname{Arg} \lambda<-\delta
\end{array}\right.
$$

$t \in[0, r]$ et $|\lambda|$ assez grand, le $O\left(\frac{e^{|I m \lambda| t}}{|\lambda|^{\frac{3}{2}}}\right)$ étant uniforme par rapport à $t \in[0, r]$. D'autre part on a. $F(a, b ; c ; z)=F(b, a ; c ; z)$ et, ainsi, (33) est valable pour:

$$
\left\{\begin{array}{c}
-\operatorname{Arctg}\left(\frac{\pi}{2 r}\right)+\delta<\operatorname{Arg} \lambda<\pi-\delta \\
\quad \text { ou bien } \\
\delta<\operatorname{Arg} \lambda<\pi+\operatorname{Arctg}\left(\frac{\pi}{2 r}\right)-\delta .
\end{array}\right.
$$

On obtient alors, par recollement des cas (34) et (35), que le développement (33) est valable dans $\mathbb{C}$ lorsque $|\lambda| \longrightarrow+\infty$ (on choisit $\left.0<\delta<\operatorname{Arctg}\left(\frac{\pi}{2 \pi}\right)\right)$.

D'après (16) on a:

$$
\begin{aligned}
\varphi_{\lambda}^{(k, k)}(r)=F(k+\nu, k+1-\nu ; & \left.k+1 ;-(s h r)^{2}\right) \\
= & \frac{1}{(\operatorname{ch} r)^{2 k}} F\left(\nu, 1-\nu ; k+1 ;-(s h r)^{2}\right) .
\end{aligned}
$$


On observe, comme il est bien connu,

$$
\frac{\Gamma(\nu)}{\Gamma(\nu+k)} \sim \frac{1}{\nu^{k}} \sim\left(\frac{2}{i}\right)^{k} \frac{1}{\lambda^{k}} \text { pour }|\lambda| \text { assez grand }
$$

par suite, on a

$$
\varphi_{\lambda}^{(k, k)}(r)=\frac{C_{k}(r)}{\lambda^{k+\frac{1}{2}}} \cos \left(\lambda r-(2 k+1) \frac{\pi}{4}\right)+O\left(\frac{e^{|I m \lambda| r}}{|\lambda|^{k+\frac{3}{2}}}\right)
$$

avec

$$
C_{k}(r)=\frac{k !}{\sqrt{\pi}}\left(\frac{2}{s h r c h r}\right)^{k+\frac{2}{2}}
$$

Exprimant, de même, $\varphi_{\lambda}^{(1,1)}\left(a_{j}\right), j=1,2$, nous en déduisons l'egalité:

$$
\begin{array}{r}
\theta(\lambda)=\frac{C_{k}}{\lambda^{k+\frac{7}{2}}} \cos \left(a_{1} \lambda-\frac{3 \pi}{4}\right) \cos \left(a_{2} \lambda-\frac{3 \pi}{4}\right) \cos \left(a_{3} \lambda-\frac{(2 k+1) \pi}{4}\right) \\
+O\left(\frac{e^{|I m \lambda|\left(a_{2}+a_{2}+a_{3}\right)}}{|\lambda|^{k+\frac{9}{2}}}\right)
\end{array}
$$

avec

$$
\begin{aligned}
C_{k}= & C_{k}\left(a_{1}, a_{2}, a_{3}\right) \\
& =\frac{k !}{(\sqrt{\pi})^{3}}\left(\frac{2}{s h a_{1} c h a_{1}}\right)^{\frac{3}{2}}\left(\frac{2}{\operatorname{sh} a_{2} c h a_{2}}\right)^{\frac{3}{2}}\left(\frac{2}{s h a_{3} c h a_{3}}\right)^{k+\frac{1}{2}}
\end{aligned}
$$

Nous allons maintenant étudier la fonction $\theta$ à travers l'étude du produit

$$
\cos \left(a_{1} \lambda-\frac{3 \pi}{4}\right) \cos \left(a_{2} \lambda-\frac{3 \pi}{4}\right) \cos \left(a_{3} \lambda-\frac{(2 k+1) \pi}{4}\right) .
$$

Soient $V=\left\{(2 l+1) \frac{\pi}{2}, l \in \mathbb{Z}\right\}$, l'ensemble des zéros de la fonction cos et $d(\lambda, V)=\operatorname{Min}(1, \operatorname{dist}(\lambda, V))$. La fonction cosinus vérifie l'inégalité globale de type Lojasiewicz:

$$
|\cos (\lambda)| \geq \frac{d(\lambda, V)}{\pi e} e^{|I m \lambda|} .
$$

Nous allons commencer par évaluer le nombre de zéros que ce produit peut possèder dans un intervalle $[l, l+1], 1$ entier. Si a est un réel $>0$ il 
y a au plus $\left[\frac{a}{\pi}\right] \leq \frac{a}{\pi}$ zéros de $\cos \left(a \lambda-\frac{(2 k+1) \pi}{4}\right)$ dans $[l, l+1]$. En effet chercher combien d'entiers $n$ sont tels que:

$$
a \lambda=\frac{(2 k+1) \pi}{4}+\frac{\pi}{2}+n \pi \in[a l, a(l+1)]
$$

revient a chercher combien de fois on trouve $\pi$ dans a. Il y a donc au plus $\left[\frac{a_{1}+a_{2}+a_{3}}{\pi}\right]$ zéros du produit ci-dessus dans $[l, l+1]$.

On partage $[l, l+1]$ en $N=\left[\frac{a_{1}+a_{2}+a_{3}}{\pi}\right]+1$ parties égales. L'une de ces parties est sans zéro. La longueur d'une telle partie étant

$$
\frac{1}{N} \geq \frac{\pi}{a_{1}+a_{2}+a_{3}+\pi},
$$

il existe un cercle de rayon $\left.\rho_{l} \in\right] l, l+1$ [ tel que la distance de $\rho_{l}$ aux zéros du produit soit au moins

$$
\frac{\pi}{2\left(a_{1}+a_{2}+a_{3}+\pi\right)} .
$$

Alors la distance de $\lambda$ tel que $|\lambda|=\rho_{l}$ aux zéros de la fonction

$$
\lambda \mapsto \cos \left(a_{j} \lambda-\frac{(2 k+1) \pi}{4}\right)
$$

est supérieure ou égale à $\frac{\pi}{2\left(a_{1}+a_{2}+a_{3}+\pi\right)}$, et ainsi, la distance de $\left(a_{j} \lambda-\frac{(2 k+1) \pi}{a_{j} \frac{4}{\pi}}\right)$ aux zéros de la fonction cos est supérieure ou égale à $\frac{a_{j} \pi}{2\left(a_{1}+a_{2}+a_{3}+\pi\right)}$. Par suite, pour $|\lambda|=\rho_{l}$, on a

$$
\begin{array}{r}
\left|\cos \left(a_{j} \lambda-\frac{(2 k+1) \pi}{4}\right)\right| \geq \frac{1}{\pi e} \operatorname{Min}\left(1, \frac{\pi a_{j}}{2\left(\pi+a_{1}+a_{2}+a_{3}\right)}\right) e^{a_{j}|I m \lambda|} \\
\geq \operatorname{Min}\left(\frac{1}{2 e\left(\pi+a_{1}+a_{2}+a_{3}\right)}, \frac{\pi a_{j}}{2 \pi e\left(\pi+a_{1}+a_{2}+a_{3}\right)}\right) e^{a_{j}|I m \lambda|} \\
\geq \frac{\operatorname{Min}\left(1, a_{j}\right)}{2 e\left(\pi+a_{1}+a_{2}+a_{3}\right)} e^{a_{3} \mid \operatorname{Im\lambda |} .}
\end{array}
$$

Par ailleur, si $|\operatorname{Im} \lambda| \geq 1$, nous avons

$$
\left|\operatorname{Im}\left(a_{j} \lambda-\frac{(2 k+1) \pi}{4}\right)\right| \geq a_{j},
$$


et la distance de $\left(a_{j} \lambda-\frac{(2 k+1) \pi}{4}\right)$ aux zéros de la fonction cos est au moins $a_{j}$ et donc

$$
\left|\cos \left(a_{j} \lambda-\frac{(2 k+1) \pi}{4}\right)\right| \geq \frac{\operatorname{Min}\left(1, a_{j}\right)}{\pi e} e^{a_{j}|\operatorname{Im} \lambda|} .
$$

D'où, pour $|\operatorname{Im} \lambda| \geq 1$ ou $|\lambda|=\rho_{l}$, on a:

$$
\left|\cos \left(a_{j} \lambda-\frac{(2 k+1) \pi}{4}\right)\right| \geq \frac{\operatorname{Min}\left(1, a_{j}\right) e^{a_{j}|m \lambda \lambda|}}{\left[2 e \pi\left(\pi+a_{1}+a_{2}+a_{3}\right)\right]} .
$$

Par suite pour $|\operatorname{Im} \lambda| \geq 1$ ou $|\lambda|=\rho_{l}$ on $a:$

$$
\begin{aligned}
\left|\cos \left(a_{1} \lambda-\frac{3 \pi}{4}\right) \cos \left(a_{2} \lambda-\frac{3 \pi}{4}\right) \cos \left(a_{3} \lambda-\frac{(2 k+1) \pi}{4}\right)\right| \\
\geq \frac{\prod_{j=1}^{j=3} \operatorname{Min}\left(1, a_{j}\right) e^{a_{j}|J m \lambda|}}{\left[2 e \pi\left(\pi+a_{1}+a_{2}+a_{3}\right)\right]^{3}} .
\end{aligned}
$$

Finalement

$$
|\theta(\lambda)| \geq \frac{C_{k}\left(a_{1}, a_{2}, a_{3}\right) \prod_{j=1}^{j=3} \operatorname{Min}\left(1, a_{j}\right) e^{a_{j}|I m \lambda|}}{\left[2 e \pi\left(\pi+a_{1}+a_{2}+a_{3}\right)\right]^{3}|\lambda|^{k+\frac{7}{2}}}-\left|O\left(\frac{e^{\left(a_{1}+a_{2}+a_{3}\right)|I m \lambda|}}{|\lambda|^{k+\frac{9}{2}}}\right)\right| .
$$

Ainsi il existe $L_{k}$ tel que pour $l \geq L_{k}$ on ait: Si $|\lambda|=\rho_{l}$ ou $|\operatorname{Im} \lambda| \geq 1$ et $|\lambda| \geq L_{k}$ alors

$$
|\theta(\lambda)| \geq \frac{A_{k}}{|\lambda|^{k+\frac{7}{2}}} e^{\left(a_{1}+a_{2}+a_{3}\right)|I m \lambda|}
$$

avec

$$
A_{k}=\frac{C_{k}\left(a_{1}, a_{2}, a_{3}\right) \prod_{j=1}^{j=3} \operatorname{Min}\left(1, a_{j}\right)}{2\left[2 e \pi\left(\pi+a_{1}+a_{2}+a_{3}\right)\right]^{3}}
$$

4.5 Lemme. Pour tout $k \in \mathbb{N}, r>0$ et $\lambda \in \mathbb{C}$ on $a$ :

$$
\left|\varphi_{\lambda}^{(k, k)}(r)\right|=\left|\varphi_{-\lambda}^{(k, k)}(r)\right| \leq \frac{k ! e^{r}}{(\operatorname{shrch} r)^{k}} \frac{e^{|I m \lambda| r}}{\left|P_{k}(i \lambda)\right|} \text { pour } \lambda \neq(2 m+1) i
$$

Preuve: D'après [7, page $60(73)]$ on a

$$
F\left(\nu, 1-\nu ; k+1 ;-(s h r)^{2}\right)=\frac{\Gamma(\nu) k !}{\Gamma(\nu+k)(t h r)^{k}} \phi_{\lambda, k}(t h r)
$$


avec

(49) $\phi_{\lambda, k}(t h r)=\int_{B} e_{i \lambda+1, b}(t h r) b^{k} d b=\int_{B}\left(\frac{1-(t h r)^{2}}{|t h r-b|^{2}}\right)^{\frac{1+i \lambda}{2}} b^{k} d b$.

On a

$$
\frac{1-(t h r)^{2}}{|t h r-b|} \leq \frac{1-(t h r)^{2}}{(1-t h r)^{2}}=e^{2 r}
$$

par suite

$$
\left|\left(\frac{1-(t h r)^{2}}{|t h r-b|^{2}}\right)^{\frac{1+i \lambda}{2}}\right| \leq e^{r} e^{|I m \lambda| r}, \quad \text { d'où }\left|\phi_{\lambda, k}(t h r)\right| \leq e^{r} e^{|I m \lambda| r} .
$$

En utilisant la relation (48) on trouve

$$
\begin{aligned}
\left|\varphi_{\lambda}^{(k, k)}(r)\right| & =\frac{1}{(c h r)^{2 k}}\left|F\left(\nu, 1-\nu ; k+1 ;-(s h r)^{2}\right)\right| \\
& =\frac{k !}{(s h r c h r)^{k}\left|P_{k}(i \lambda)\right|}\left|\phi_{\lambda_{,} k}(t h r)\right| \\
& \leq \frac{k ! e^{r}}{(s h r c h r)^{k}} \frac{e^{|I m \lambda| r}}{\left|P_{k}(i \lambda)\right|} \cdot \mathbf{a}
\end{aligned}
$$

4.6 Lemme. Soit $f$ une fonction $C^{\infty}$ dans $B(0, R)$ et, pour choque $0<r<R, z=\rho e^{i \varphi}, \rho=t h r$

$$
f(z)=f\left(\rho e^{i \varphi}\right)=\sum_{k \in \mathbb{Z}} a_{k}(\rho) e^{i k \varphi}
$$

le développement de Fourier de la fonction $2 \pi$-periodique $\theta \longmapsto f\left(\rho e^{i \theta}\right)$, évaluée en $\theta=\varphi$.

On a

(i) pour $k \neq 0 a_{k}(\rho)=\frac{1}{\rho^{|k|} \mid \pi(c h r)^{2}(|k|-1) !}\left\langle S_{k} \times T_{\rho, k}, f\right\rangle$

(ii) pour $k=0 \quad a_{0}(\rho)=\langle\chi \partial B(0, r), f\rangle$.

Preuve:

$$
\begin{aligned}
a_{k}(\rho) & =\frac{1}{2 \pi} \int_{0}^{2 \pi} f\left(\rho e^{i \theta}\right) e^{-i k \theta} d \theta \\
& = \begin{cases}\frac{1}{\rho^{k}} \int_{|w|=\rho} f(w)(\bar{w})^{k} d \sigma_{\rho}(w) & \text { si } k>0 \\
\frac{1}{\rho^{-k}} f_{|w|=\rho} f(w)(w)^{-k} d \sigma_{\rho}(w) & \text { si } k<0\end{cases} \\
& =\frac{1}{\rho^{|k|}} \int_{|w|=\rho} f(w) H_{k}(w) d \sigma_{\rho}(w)
\end{aligned}
$$


pour tout $k \in \mathbb{Z}$, ce que l'on peut écrire

$$
a_{k}(\rho)=\frac{1}{\rho^{|k|}}<H_{k} \cdot \sigma_{r}, f>.
$$

Or, d'après le lemme 4.3 , nous avons

$$
H_{k} \sigma_{r}=\frac{I}{\pi(\operatorname{chr} r)^{2}(|k|-1) !} S_{k} \times T_{\rho, k} \text { pour } k \neq 0
$$

d'où le lemme.

\section{Reconstruction de $f$}

Nous suivons la méthode de [3]. L'idée de base consiste à construire deux suites de distributions radiales $\mu_{l}=\mu_{l}(r, k)$ et $\nu_{l}=\nu_{l}(r, k)$, éléments de $\mathcal{E}_{0}^{\prime}(D)$ pour tout $l \geq 1$ telles que:

$$
T_{\rho, k}=\lim _{l \rightarrow \infty}\left[\mu_{l} \times \chi_{B\left(0, r_{1}\right)}+\nu_{l} \times \chi_{B\left(0, r_{2}\right)}\right]
$$

car, alors, on sera capable de restituer les coefficients $a_{k}(\rho)$ du développement en série de Fourier de $f$, conformément à

$$
a_{k}(\rho)=\lim _{l \rightarrow \infty} \frac{1}{\rho^{|k|} \pi(c h r)^{2}(|k|-1) !}\left[\left\langle\mu_{l}, \check{S}_{k} \times f \times \check{\chi_{1}}\right\rangle+\left\langle\nu_{l}, \check{S_{k}} \times f \times \check{\chi_{2}}\right\rangle\right]
$$

en utilisant la connaissance des "moyennes", $f \times \ddot{\chi}_{1}$ et $f \times \ddot{\chi}_{2}$. Pour cela nous introduisons une suite strictement croissante: $0=R_{0}<r_{1}+r_{2}<$ $R_{1}<R_{2}<\cdots<R_{p} \nearrow R$. et une suite $\left(\varepsilon_{p}\right)_{p \geq 1}$ strictement croissante telle que: $R_{p}=\left(r_{1}+r_{2}\right)\left(1+\varepsilon_{p}\right)$, en exigeant de plus que

$$
\lim _{p \rightarrow+\infty} \frac{R-R_{p-1}}{R-R_{p}}=1
$$

On pose $\beta_{p}=\varepsilon_{p}\left(r_{1}+r_{2}\right)$.

La décomposition de $T_{\rho, k}$ va dépendre de la valeur de l'entier $p$ tel que $r \in\left|R_{p-1}, R_{p}\right|$. La transformée sphérique de $T_{\rho, k}\left(\rho=t h r, k \in \mathbb{N}^{*}\right)$ est:

$$
\widetilde{T}_{\rho, k}=\frac{\pi}{k}(c h r)^{2}(s h r)^{2 k} \varphi_{\lambda}^{(k, k)}(r)=\pi(k-1) !(c h r)^{2}(s h r)^{2 k} G_{k}(\lambda, r)
$$

avec

$$
G_{k}(\lambda, r)=\frac{1}{k !} \varphi_{\lambda}^{(k, k)}(r)
$$


5.1 Proposition. Soient $r_{1}, r_{2}>0,\left(r_{1}+r_{2}<R\right)$ tels que les équations:

$$
\left\{\begin{array}{l}
\varphi_{\lambda}^{(1,1)}\left(r_{1}\right)=0 \\
\varphi_{\lambda}^{(1,1)}\left(r_{2}\right)=0,
\end{array}\right.
$$

n'aient aucune solution commune $\lambda \in \mathbb{C}$. Pour tout $k, p \in \mathbb{N}, r \in$ $\left[R_{p-1}, R_{p}[\right.$, il existe deux suites de distributions radiales (explicitables) $\mu_{l}, \nu_{l}(l \geq 1)$ vérifant:

$$
\begin{aligned}
\left|G_{k}(\lambda, r)-\left(G_{1}\left(\lambda, r_{1}\right) \widetilde{\mu}_{l}(\lambda)+G_{1}\left(\lambda, r_{2}\right) \widetilde{\nu}_{l}(\lambda)\right)\right| \\
\quad \leq \frac{c^{\prime}}{l}(\text { shrchr })^{-k} \frac{|| \lambda||^{3} e^{R_{p}|I m \lambda|}}{\left|P_{k}(i \lambda)\right|}
\end{aligned}
$$

pour $l \geq \max \left(L_{k}, k\right)$ et $\|\lambda\|=\max (1,|\lambda|)$,

$$
\begin{aligned}
& \left|\tilde{\mu}_{l}(\lambda)\right| \leq c^{\prime \prime}(\operatorname{shrch} r)^{-k} \frac{\|\left.\lambda\right|^{3} e^{\left(r_{2}+\beta_{p}\right)|I m \lambda|}}{\left|P_{k}(i \lambda)\right|} \\
& \left|\tilde{\nu}_{l}(\lambda)\right| \leq c^{\prime \prime}(\operatorname{shrch} r)^{-k} \frac{\|\left.\lambda\right|^{3} e^{\left(r_{1}+\beta_{p}\right)|I m \lambda|}}{\left|P_{k}(i \lambda)\right|}
\end{aligned}
$$

avec $c^{\prime}$ une constante strictement positive dépendant de $r_{1}, r_{2}, R$, et $\varepsilon_{1}$. La constante $c^{\prime \prime}$ depend de plus aussi de l et de $k$.

Preuve: Nous allons appliquer le lemme 4.3 avec $a_{1}=r_{1}, a_{2}=r_{2}$ et $a_{3}=\varepsilon_{p}\left(r_{1}+r_{2}\right)$.

Posons pour la suite

$$
\left\{\begin{array}{l}
\Theta(\lambda)=G_{1}\left(\lambda, r_{1}\right) G_{1}\left(\lambda, r_{2}\right) G_{k}\left(\lambda, \beta_{p}\right) \\
g(\lambda)=G_{k}(\lambda, r) .
\end{array}\right.
$$

L'idée de la preuve consiste à utiliser la relation

$$
\frac{1}{2 i \pi} \int_{|\zeta|=\rho_{l}} \frac{g(\zeta)}{\zeta-\lambda} d \zeta= \begin{cases}g(\lambda) & \text { si }|\lambda|<\rho_{l} \\ 0 & \text { si }|\lambda|>\rho_{l}\end{cases}
$$

Soit $q$ un entier à fixer plus tard. La relation (60) peut aussi s'écrire sous la forme:

$$
\begin{aligned}
& \frac{1}{2 i \pi} \int_{|\zeta|=\rho_{l}} \frac{g(\zeta)}{\zeta^{q} \Theta(\zeta)} \frac{\zeta^{q} \Theta(\zeta)-\lambda^{q} \Theta(\lambda)}{\zeta-\lambda} d \zeta \\
& \quad+\frac{1}{2 i \pi} \lambda^{q} \Theta(\lambda) \int_{|\zeta|=\rho_{l}} \frac{g(\zeta)}{\zeta^{q} \Theta(\zeta)} \frac{d \zeta}{\zeta-\lambda}= \begin{cases}g(\lambda) & \text { si }|\lambda|<\rho_{l} \\
0 & \text { si }|\lambda|>\rho_{l}\end{cases}
\end{aligned}
$$


On introduit la fonction entière paire

$$
H_{l}(\lambda)=\frac{1}{2 i \pi} \int_{|\zeta|=\rho_{l}} \frac{g(\zeta)}{\zeta^{q} \Theta(\zeta)} \frac{\zeta^{q} \Theta(\zeta)-\lambda^{q} \Theta(\lambda)}{\zeta-\lambda} d \zeta
$$

C'est l'interpolation de $g$ sur les zéros de la fonction $\lambda^{q} \Theta(\lambda)$. La parité de $H_{l}(\lambda)$ est indépendante de celle de $q$. On peut choisir la suite $\left(\varepsilon_{p}\right)_{p \geq 1}$ pour que les couples de fonctions

$$
\begin{cases}G_{1}\left(\lambda, r_{1}\right) & \text { et } G_{k}\left(\lambda, \beta_{p}\right) \\ G_{1}\left(\lambda, r_{2}\right) & \text { et } G_{k}\left(\lambda, \beta_{p}\right)\end{cases}
$$

soient sans zéros communs dans $\mathbb{C}$, en effet soit $A_{i}=\left\{\lambda_{j}^{i}, j \in \mathbb{N}\right\}$ l'ensemble des zéros de la fonction $\lambda \longmapsto G\left(\lambda, r_{i}\right)$. Pour tout $j, k \in \mathbb{N}$ on désigne par $A_{i, j, k}$ l'ensemble dénombrable des zeros, dans $] 0,+\infty$, de la fonction $r \longmapsto G_{k}\left(\lambda_{j}^{i}, r\right)$, qui est réelle analytique et non nulle $\left(G_{k}\left(\lambda_{j}^{i}, 0\right) \neq 0\right)$. Il suffit alors de choisir la suite $\left(\varepsilon_{p}\right)_{p \geq 1}$ telle que pour tout $p \in \mathbb{N}$

$$
\beta_{p} \notin \bigcup_{j, k \in \mathbb{N}}\left(A_{1, j, k} \cup A_{2, j, k}\right) .
$$

Le théorème des résidus donne:

$$
\begin{array}{r}
H_{l}(\lambda)=\lambda^{q} G_{1}\left(\lambda, r_{1}\right) G_{1}\left(\lambda, r_{2}\right) g_{3, l}(\lambda)+\lambda^{q} G_{1}\left(\lambda, r_{1}\right) G_{k}\left(\lambda, \beta_{p}\right) g_{2, l}(\lambda) \\
+\lambda^{q} G_{1}\left(\lambda, r_{2}\right) G_{k}\left(\lambda, \beta_{p}\right) g_{1, l}(\lambda)+\Theta(\lambda) P(\lambda)
\end{array}
$$

avec

$$
g_{1, l}(\lambda)=\sum_{\substack{G_{1}\left(\alpha, r_{1}\right)=0 \\|\alpha|<\rho_{l}}} \frac{G_{1}\left(\lambda, r_{1}\right)}{(m(\alpha)-1) !} \frac{d^{m(\alpha)-1}}{d \zeta^{m(\alpha)-1}}\left[\frac{g(\zeta)(\zeta-\alpha)^{m(\alpha)}}{\zeta^{q \Theta(\zeta)(\lambda-\zeta)}}\right]_{\zeta=\alpha}
$$

(66)

$$
g_{2, l}(\lambda)=\sum_{\substack{G_{2}\left(\alpha, r_{2}\right)=0 \\|\alpha|<\rho_{l}}} \frac{G_{2}\left(\lambda, r_{2}\right)}{(m(\alpha)-1) !} \frac{d^{m(\alpha)-1}}{d \zeta^{m(\alpha)-1}}\left[\frac{g(\zeta)(\zeta-\alpha)^{m(\alpha)}}{\zeta^{q \Theta(\zeta)(\lambda-\zeta)}}\right]_{\zeta=\alpha}
$$

$$
g_{3, l}(\lambda)=\sum_{\substack{G_{k}\left(\alpha, \beta_{p}\right)=0 \\|\alpha|<p_{l}}} \frac{G_{k}\left(\lambda, \beta_{p}\right)}{(m(\alpha)-1) !} \frac{d^{m(\alpha)-1}}{d \zeta^{m(\alpha)-1}}\left[\frac{g(\zeta)(\zeta-\alpha)^{m(\alpha)}}{\zeta \Theta(\zeta)(\lambda-\zeta)}\right]_{\zeta=\alpha}
$$

$$
P(\lambda)=\operatorname{Res}\left(\frac{g(\zeta)\left(\lambda^{q-1}+\zeta \lambda^{q-2}+\cdots+\zeta^{q-1}\right)}{\zeta^{q} \Theta(\zeta)}, 0\right),
$$


où $m(\alpha)$ est la multiplicité de $\alpha$. Il est clair d'après (37) que $m(\alpha)=1$ pour $|\lambda|$ assez grand comme on le voit en utilisant le théorème de Rouché. Nous ne savons pas si $m(\alpha)$ est constament égale à 1 pour les valeurs petites de $|\lambda|$.

On observe que sí $\alpha$ est un zeró de $\lambda \longmapsto \lambda^{q} \Theta(\lambda)$ on a:

$$
H_{l}(\alpha)= \begin{cases}g(\alpha) & \text { si }|\alpha|<\rho_{l} \\ 0 & \text { si }|\alpha|>\rho_{l}\end{cases}
$$

car

$$
H_{l}(\lambda)+\frac{1}{2 i \pi} \lambda^{q} \Theta(\lambda) \int_{1 \zeta \mathrm{i}=\rho_{l}} \frac{g(\zeta)}{\zeta^{q} \Theta(\zeta)} \frac{d \zeta}{\zeta-\lambda}= \begin{cases}g(\lambda) & \text { si }|\lambda|<\rho_{l} \\ 0 & \text { si }|\lambda|>\rho_{l}\end{cases}
$$

Les distributions $\mu_{l}$ et $\nu_{l}$ seront alors défnies par:

$$
\left\{\begin{array}{l}
\tilde{\mu}_{l}(\lambda)=\lambda^{q} G_{1}\left(\lambda, r_{2}\right) g_{3, l}(\lambda)+\lambda^{q} G_{k}\left(\lambda, \beta_{p}\right) g_{2, l}(\lambda) \\
\tilde{\nu}_{l}(\lambda)=\lambda^{q} G_{k}\left(\lambda, \beta_{p}\right) g_{1, l}(\lambda)+P(\lambda) G_{1}\left(\lambda, r_{1}\right) G_{k}\left(\lambda, \beta_{p}\right)
\end{array}\right.
$$

et ceci nous foumira la factorisation suivante:

$$
H_{l}(\lambda)=\widetilde{\mu}_{l}(\lambda) G_{1}\left(\lambda, r_{1}\right)+\widetilde{\nu}_{l}(\lambda) G_{1}\left(\lambda, r_{2}\right)
$$

et les estimations sur $\left|\tilde{\mu}_{l}\right|$ et $\left|\tilde{\nu}_{l}\right|$.

Nous allons commencer par montrer que la famille $\left(H_{l}\right)_{l \geq L_{k}}$ est bornée dans $\widehat{\mathcal{E}^{\prime}(D)}$.

Nous disposons des inégalités suivantes: (lemme 4.4)

$$
\begin{aligned}
|g(\lambda)| & \leq \frac{e^{T}}{(\operatorname{shrch} r)^{k}} \frac{e^{|I m \lambda| r}}{\left|P_{k}(i \lambda)\right|} \\
|\Theta(\lambda)| & \leq \frac{e^{R_{p}}}{\left(\operatorname{sh} r_{1} c h r_{1}\right)\left(\operatorname{sh} r_{2} \operatorname{ch} r_{2}\right)\left(\operatorname{shr} r_{\beta_{p}} \operatorname{ch} r_{\beta_{p}}\right)^{k}} \frac{e^{|I m \lambda| R_{p}}}{\left|P_{1}(i \lambda)\right|^{2}\left|P_{k}(i \lambda)\right|},
\end{aligned}
$$

les constantes $e^{T}$ et $e^{R_{p}}$ peuvent étre remplacer par $e^{R}$. On choisit $l \geq$ $\max \left(L_{k}, k\right) \geq 2$, pour $|\lambda|=\rho_{l}$. D'après (29) on a:

$$
|\Theta(\lambda)| \geq \frac{c_{1} 2^{k}}{\left(s h r_{1} c h r_{1}\right)^{\frac{3}{2}}\left(s h r_{2} c h r_{2}\right)^{\frac{3}{2}}\left(\operatorname{sh} \beta_{p} \operatorname{ch} \beta_{p}\right)^{k+\frac{1}{2}}} \frac{e^{|I m \lambda| R_{p}}}{\rho_{l}^{k+\frac{7}{2}}}
$$

avec

$$
c_{1}=c_{1}\left(r_{1}, r_{2}, R\right)=\frac{1}{\sqrt{2}(\sqrt{\pi})^{3}} \frac{\left[\min \left(1, r_{1}, r_{2}, \beta_{p}\right)\right]^{3}}{[e \pi(\pi+R)]^{3}} .
$$


De plus on remarque que si $\operatorname{Re} \lambda \geq 0$ et $\lambda \neq(2 m+1) i m \in \mathbb{N}$ on a:

$$
\left|P_{k}(\lambda)\right| \geq\left(\frac{|\lambda|}{2}\right)^{k}
$$

Car, pour tout $k \in \mathbb{N}$ et $z \in \mathbb{C}$ tel que Re $z \geq 0$, on a $|z+k| \geq|z|$. Comme la fonction $g$ est paire on peut supposer que $\operatorname{Re}(i \lambda) \geq 0$. Par suite (72) s'écrit

$$
|g(\lambda)| \leq \frac{2^{k} e^{r}}{(\operatorname{shrch} r)^{k}} \frac{e^{|I m \lambda| r}}{|\lambda|^{k}} .
$$

Pour estimer $H_{l}(\lambda)$ lorsque $|\lambda|>\rho_{l}$ nous utiliserons l'éxpression:

$$
H_{l}(\lambda)=-\frac{1}{2 i \pi} \lambda^{q} \Theta(\lambda) \int_{|\zeta|=\rho_{l}} \frac{g(\zeta)}{\zeta^{q} \Theta(\zeta)} \frac{d \zeta}{\zeta-\lambda}
$$

Les relations (74) et (76) donnent

$$
\begin{aligned}
\left|H_{l}(\lambda)\right| & \leq \frac{e^{T}\left(\operatorname{sh} r_{1} \operatorname{ch} r_{1}\right)^{\frac{3}{2}}\left(\operatorname{sh} r_{2} \operatorname{ch} r_{2}\right)^{\frac{3}{2}}\left(\operatorname{sh} \beta_{p} \operatorname{ch} \beta_{p}\right)^{k+\frac{3}{2}}}{c_{1}(\operatorname{shrch} r)^{k} \rho_{l}^{q-\frac{9}{2}}} \frac{|\lambda|^{q}|\Theta(\lambda)|}{|\lambda|-\rho_{l}} \\
& \leq \frac{e^{r}\left(s h r_{1} \operatorname{ch} r_{1}\right)^{\frac{3}{2}}\left(\operatorname{sh} r_{2} \operatorname{ch} r_{2}\right)^{\frac{3}{2}}\left(\operatorname{sh} \beta_{p} \operatorname{ch} \beta_{p}\right)^{k+\frac{1}{2}}}{c_{1}(\operatorname{shr} r h)^{k}} \frac{|\lambda|^{5}|\Theta(\lambda)|}{|\lambda|-\rho_{l}},
\end{aligned}
$$

avec $q=5$.

D'après (73) et $(75)$ on a:

$$
\left|H_{l}(\lambda)\right| \leq \frac{c_{2}}{(\operatorname{shrch} r)^{k}} \frac{|\lambda|^{3} e^{R_{p}|I m \lambda|}}{\left|P_{k}(i \lambda)\right|\left\{|\lambda|-\rho_{l}\right)},
$$

avec

$$
\begin{aligned}
c_{2}=c_{2}\left(r_{1}, r_{2}, R\right) \leq \frac{4 e^{2 R}}{c_{1}}\left(s h r_{1} c h r_{1}\right)^{\frac{1}{2}}\left(s h r_{2} \operatorname{ch} r_{2}\right)^{\frac{1}{2}}\left(\operatorname{sh} \beta_{p} \operatorname{ch} \beta_{p}\right)^{\frac{1}{2}} & \\
& \leq c_{2}^{\prime}\left(r_{1}, r_{2}, R\right)=c_{2}^{\prime} .
\end{aligned}
$$

Pour estimer $H_{l}(\lambda)$ Iorsque $|\lambda|<\rho_{l}$ nous utiliserons cette fois-ci l'expression

$$
H_{l}(\lambda) \mid=g(\lambda)-\frac{1}{2 i \pi} \lambda^{5} \Theta(\lambda) \int_{|\zeta|=\rho_{l}} \frac{g(\zeta)}{\zeta^{5} \Theta(\zeta)} \frac{d \zeta}{\zeta-\lambda}
$$


Il vient

$$
\begin{aligned}
\left|H_{l}(\lambda)\right| & \leq|g(\lambda)|+\frac{c_{2}}{(s h r c h r)^{k}} \frac{|\lambda|^{3} e^{R_{p}|I m \lambda|}}{\left|P_{k}(i \lambda)\right|\left(\rho_{l}-|\lambda|\right)} \\
& \leq \frac{e^{r}}{(s h r c h r)^{k}} \frac{e^{R_{p}|I m \lambda|}}{\left|P_{k}(i \lambda)\right|}+\frac{c_{2}}{(s h r c h r)^{k}} \frac{|\lambda|^{3} e^{R_{p}|I m \lambda|}}{\left|P_{k}(i \lambda)\right|\left(\rho_{l}-|\lambda|\right)} \\
& \leq \frac{c_{2}^{\prime}}{(s h r c h r)^{k}} \frac{e^{R_{p}|I m \lambda|}}{\left|P_{k}(i \lambda)\right|}\left(1+\frac{|\lambda|^{3}}{\rho_{l}-|\lambda|}\right) .
\end{aligned}
$$

On déduit de cette dernière inégalité que, pour $|\lambda| \leq l-1$ on a (car $\left.\rho_{l}-|\lambda| \geq 1\right)$

$$
\left|H_{l}(\lambda)\right| \leq \frac{2 c_{2}^{\prime}}{(\operatorname{shrchr} r)^{k}} \frac{\|\left.\lambda\right|^{3} e^{R_{\mathrm{p}}|I m \lambda|}}{\left|P_{k}(i \lambda)\right|} \text { avec } \| \lambda||=\max (1,|\lambda|),
$$

d'où, si $|\lambda| \notin[l-1, l+2]$, puisque ||$\lambda\left|-\rho_{l}\right| \geq 1$, on a:

$$
\left|H_{l}(\lambda)\right| \leq \frac{2 c_{2}^{\prime}}{(\operatorname{shrchr})^{k}} \frac{\|\lambda\|^{3} e^{R_{p}|\operatorname{Im} \lambda|}}{\left|P_{k}(i \lambda)\right|} .
$$

Dans la couronne $l-1 \leq|\lambda| \leq l+2$, le principe de maximum donne :

$$
\left|H_{l}(\lambda)\right| \leq \frac{c_{2}^{\prime \prime}}{(\operatorname{shrch} r)^{k}} \frac{\|\left.\lambda\right|^{3} e^{R_{p}|\lambda|}}{\left|P_{k}(i \lambda)\right|} .
$$

La constante $c_{2}^{\prime \prime}$ étant indépendante de $k$. On déduit de (79) et (80) que (80) est exacte dans $\mathbb{C}$ en modifiant la constarte $c_{2}^{\prime \prime}$ éventueliement.

On conclut alors que $\left(H_{l}\right)_{l \geq 1}$ est un ensemble borné dans

$$
\operatorname{Exp}(\mathbb{C})=\underset{N>0}{\lim } \operatorname{Exp}(\mathbb{C})
$$

avec

$$
\operatorname{Exp}_{N}(\mathbb{C})=\left\{f \in \mathcal{H}(\mathbb{C}): \sup _{\lambda \in \mathbb{C}} e^{-N|\lambda|}|f(\lambda)|=\|f\|_{N}<\infty\right\}
$$

Ce que nous voulons montrer est que, en fait, $\left(H_{l}\right)_{l \geq 1}$ est un ensemble borné dans $\widehat{\mathcal{E}^{\prime}(D)}$ : c'est-a dire qu'il existe $A>0$ tel que

$$
\left|H_{l}(\lambda)\right| \leq A(1+|\lambda|)^{A} e^{A|\operatorname{Im} \lambda|}, \quad \lambda \in \mathbb{C} .
$$

Nous allons d'abord estimer $H_{l}(\lambda)$ pour $\lambda=x \in \mathbb{R}$. 
Nous savons déjà que, pour $|x| \notin[l-1, l+2]$,

$$
\left|H_{l}(x)\right| \leq \frac{2 c_{2}^{\prime}}{(\text { shrchr })^{k}} \frac{\|x\|^{3} e^{R_{p}}}{\left|P_{k}(i x)\right|} .
$$

Pour $x \in[l-1, l+2]$ nous écrivons

$$
H_{l}(x)=H_{l}(x)-H_{l+3}(x)+H_{l+3}(x)
$$

avec

$$
\left|H_{l+3}(x)\right| \leq \frac{2 c_{2}^{\prime}}{(\operatorname{shrch} r)^{k}} \frac{\|x\|^{3} e^{R_{p}}}{\left|P_{k}(i x)\right|} \text {, si }|x| \in[l-1, l+2],
$$

il suffit donc d'estimer $\left(H_{l}-H_{l+3}\right)(x)$.

Soit $\Gamma_{l}$ le bord du domaine défini par: $\left\{\operatorname{Re} \lambda \geq 0, \rho_{l} \leq|\lambda| \leq\right.$ $\rho_{l+1}$ et $\left.|\operatorname{Im} \lambda| \leq 1\right\}$. Avec un choix convenable de l'orientation on a:

$$
H_{l}(x)-H_{l+3}(x)=\frac{1}{2 i \pi}\left(\int_{\Gamma_{i}}+\int_{\Gamma_{i}^{\prime}}\right) \frac{g(\zeta)}{\zeta^{5} \Theta(\zeta)} \frac{\zeta^{5} \Theta(\zeta)-x^{5} \Theta(x)}{\zeta-x} d \zeta
$$

pour $\zeta \in \Gamma_{l}$ et $l-1 \leq x \leq l+2$

$$
\begin{aligned}
\frac{\mid \zeta^{5} \Theta(\zeta)-x^{5} \Theta(x)}{|\zeta-x|} & \leq \max _{|w-x| \leq 5}\left|\frac{d}{d w}\left(w^{5} \Theta(w)\right)\right| \\
& \leq \frac{c_{3}^{\prime}}{\left(\operatorname{shr_{1}} \operatorname{ch} r_{1}\right)\left(\operatorname{sh} r_{2} \operatorname{ch} r_{2}\right)\left(\operatorname{sh} \beta_{p} \operatorname{ch} \beta_{p}\right)^{k}} \frac{\|x\|^{3}}{\left|P_{k}(i x)\right|}
\end{aligned}
$$

avec une constante $c_{3}^{\prime}$ indépendante de $k$.

Pour $\zeta \in \Gamma_{l}^{\prime}$ et $l-1 \leq x \leq l+2$

$$
\begin{aligned}
\left|\zeta^{5} \Theta(\zeta)-x^{5} \Theta(x)\right| & \leq\left|\zeta^{5} \Theta(\zeta)\right|+\left|x^{5} \Theta(x)\right| \\
& \leq \frac{2 c_{3}^{\prime}}{\left(s h r_{1} \operatorname{ch} r_{1}\right)\left(\operatorname{shr} r_{2} \operatorname{ch} r_{2}\right)\left(\operatorname{sh} \beta_{p} \operatorname{ch} \beta_{p}\right)^{k}} \frac{\left.\|x\|\right|^{3}}{\left|P_{k}(i x)\right|}
\end{aligned}
$$

ainsi pour $|x| \in[l-1, l+2]$ on a

$$
\left|H_{l}(x)\right| \leq \frac{c_{3}^{\prime \prime}}{(\operatorname{shrch} r)^{k}} \frac{1}{\|x\|^{-3}\left|P_{k}(i x)\right|}
$$

avec $c_{3}^{\prime \prime}$ indépendante de $k$.

Finalement une telle inégalité est vraie pour tout $x \in \mathbb{R}$, avec une constante au numérateur indépendante de $k$. Pour avoir des inégalités 
dans $\mathbb{C}$ nous utiliserons le principe de Phragmen-Lindelöf, dans chacun des quadrants $\operatorname{Re} \lambda \gtrless 0, \operatorname{Im} \lambda \gtrless 0$. Par exemple, dans $\operatorname{Re} \lambda \geq 0, \operatorname{Im} \lambda \geq$ 0 , nous considérons la fonction holomorphe.

$$
g_{l}(\lambda)=\left(\lambda+\frac{i}{k}\right)^{-3} P_{k}(i \lambda) H_{l}(\lambda) e^{i R_{p} \lambda} .
$$

Pour $\lambda=x \geq 0$ nous avons d'après (83) avec une constante $c$, indépendante de $k$ et de $l$, de la forme, $\frac{\widetilde{c}}{(\operatorname{shrchr} r)^{k}}$, où $\widetilde{c}$ est indépendante de $k$,

$$
\left|g_{l}(x)\right| \leq c \frac{\|x\|^{3}}{\left(\left(x+\frac{1}{k}\right)^{2}+\frac{1}{k^{2}}\right)^{3}} .
$$

Pour $\lambda=i y$, nous avons d'après (80) et toujours avec comme précédemment,

$$
\left|g_{l}(i y)\right| \leq c\left(y+\frac{1}{k}\right)^{-3}\|y\|^{3}
$$

On en déduit que $g_{l}$ reste bornée par une constante indépendante de $l$ et de $k$ dans le quadrant $\operatorname{Re} \lambda \geq 0, \operatorname{Im} \lambda \geq 0$ et donc

$$
\left|H_{l}(\lambda)\right| \leq c \frac{\|y\|^{3} e^{R_{p} I m|\lambda|}}{\left|P_{k}(i \lambda)\right|}
$$

On traite de même le cas des trois autres quadrants.

Ainsi $\left(H_{l}\right)_{l \geq 1}$ est bornée dans $\widehat{\mathcal{E}_{0}^{\prime}(D)}$.

Montrons à présent que:

$$
\lim _{l \rightarrow \infty} H_{l}(\lambda)=g(\lambda)
$$

sur tout compact comme conséquence de l'inégalité suivante, valable pour $|\lambda|<\rho_{l}$

$$
\left|H_{l}(\lambda)-g(\lambda)\right| \leq c_{2} \frac{\|\left.\lambda\right|^{3} e^{R_{p}|I m \lambda|}}{(\operatorname{shrchr} r)^{k}\left|P_{k}(i \lambda)\right|\left(\rho_{l}-|\lambda|\right)} .
$$

Si $|\lambda| \leq \frac{l}{2}$ on a

$$
\left|H_{l}(\lambda)-g(\lambda)\right| \leq \frac{c_{2}}{l} \frac{\|\lambda\|^{3} e^{R_{p}|\operatorname{Im} \lambda|}}{(\operatorname{shrchr})^{k}\left|P_{k}(i \lambda)\right|} .
$$


Si $|\lambda| \geq \frac{l}{2}$ on a

$$
\begin{aligned}
\left|H_{l}(\lambda)-g(\lambda)\right| & \leq\left|H_{l}(\lambda)\right|+|g(\lambda)| \\
& \leq \frac{2 c_{2}}{l} \frac{\|\left.\lambda\right|^{3} e^{R_{p}|I m \lambda|}}{(\operatorname{shrchr})^{k} \mid P_{k}\left(i \lambda \mid\left(\rho_{l}-|\lambda|\right)\right.} .
\end{aligned}
$$

Ainsi, pour $|\lambda|<\rho_{l}$

$$
\left|H_{l}(\lambda)-g(\lambda)\right| \leq \frac{c_{4}}{l} \frac{\|\lambda\|^{3} e^{R_{p}|\operatorname{Im} \lambda|}}{(\operatorname{shrch} r)^{k}\left|P_{k}(i \lambda)\right|}
$$

ce qui acheve la preuve de (56). les estimations (57) et (58) s'en déduisent.

Nous sommes ainsi en mesure d'énoncer:

5.2 Théorème. Soient $0<r_{1}<r_{2}\left(r_{1}+r_{2}<R\right)$, tels que les équations

$$
\varphi_{\lambda}^{(1,1)}\left(r_{j}\right)=0, j=1,2
$$

n'aient aucune solution commune $\lambda \in \mathbb{C}$ et $\left(\varepsilon_{p}\right)_{p \geq 1}$ une suite strictement croissante de réels positifs et de limite $\frac{R}{r_{1}+r_{2}}-1, R_{p}=\left(r_{1}+r_{2}\right)(1+$ $\left.\varepsilon_{p}\right), p \geq 1, R_{0}=0$, et telle que

$$
\lim _{p \rightarrow+\infty} \frac{R-R_{p-1}}{R-R_{p}}=1 .
$$

Pour tout $p \geq 1, r \in\left[R_{p-1}, R_{p} \mid\right.$ et $k \in \mathbb{Z}^{*}$ il existe deux suites de distributions $\left(\mathcal{U}_{l}\right),\left(\mathcal{B}_{l}\right)$ d'ordre $\leq 5$ a support compact dans $B(0, R-$ $\left.r_{1}\right), B\left(0, R-r_{2}\right)$ respectivement telles que pour $l \geq \max \left(L_{k}, k\right)$ et $f \in$ $C^{\infty}(B(0, R))$ on ait:

$$
\begin{aligned}
\mid \frac{1}{2 \pi} \int_{0}^{2 \pi} f\left(\rho e^{i \theta}\right) e^{-i k \theta} d \theta- & \left\langle\mathcal{U}_{l}, f \times \check{\mu}_{r_{1}}\right\rangle-\left\langle\mathcal{B}_{l}, f \times \check{\mu}_{r_{2}}\right\rangle \mid \\
& \leq \frac{\gamma}{l} \frac{1}{(R-r)^{7}} \sup _{\substack{z \in B\left(0, R_{p}^{\prime}\right) \\
0 \leq i, j \leq 3}}\left|\frac{\partial^{i+j}}{\partial z^{i} \partial \bar{z}^{j}} f(z)\right|
\end{aligned}
$$

où $\mu_{r_{j}}=\chi_{B\left(0, r_{j}\right)}, j=1,2, \rho=$ th $r, R_{p}^{\prime}=\frac{2}{3} R+\frac{1}{3} R_{p}$ et $\gamma=$ $\gamma\left(r_{1}, r_{2}, R, \varepsilon_{1}\right)>0$.

Preuve: Pour tout $k \in \mathbb{Z}^{*}$ on a

$$
a_{k}(\rho)=\frac{1}{\left.\rho^{|k|}\right|_{\pi(c h r)^{2}(|k|-1) !}}\left\langle S_{k} \times T_{\rho, k}, f\right\rangle
$$


La transformée sphérique de $T_{\rho, k}$ est:

$$
\lambda \longmapsto \pi(|k|-1) !(c h r)^{2}(s h r)^{2|k|} G_{|k|}(\lambda, r) .
$$

On applique la proposition 5.1. On peut trouver deux distributions explicites, radiales, $\mu_{l}$ et $\nu_{l}$ telles que $\operatorname{supp}\left(\mu_{l}\right) \subset B\left(0, R_{p}-r_{1}\right), \operatorname{supp}\left(\nu_{l}\right) \subset$ $B\left(0, R_{p}-r_{2}\right)$, et, telles que, si $\mathcal{T}_{l}$ est la distribution radiale de transformée sphérique:

$$
\tilde{\mathcal{T}}_{l}(\lambda)=(\operatorname{shrch} r)^{k}\left[G_{|k|}(\lambda, r)-G_{1}\left(\lambda, r_{1}\right) \tilde{\mu}_{l}(\lambda)-G_{1}\left(\lambda, r_{2}\right) \tilde{\nu}_{l}(\lambda)\right],
$$

on ait l'inégalité:

$$
\left|\tilde{\mathcal{T}}_{l}(\lambda)\right| \leq \frac{c^{\prime}}{l} \frac{\|\left.\lambda\right|^{3} e^{R_{\mathrm{p}}|I m \lambda|}}{\left|P_{k}(i \lambda)\right|}, \quad \text { pour } l \geq \max \left(L_{k}, k\right),
$$

où la constante $c^{\prime}$ est celle qui apparait dans 4.1. On définit les distributions:

$$
\begin{aligned}
& \mathcal{U}_{l}=\frac{(s h r c h r)^{|k|}}{\pi\left(c h r_{1}\right)^{2}\left(s h r_{1}\right)^{2}} S_{k} \times \mu_{l} \\
& \mathcal{B}_{l}=\frac{(s h r c h r)^{|k|}}{\pi\left(c h r_{2}\right)^{2}\left(s h r_{2}\right)^{2}} S_{k} \times \nu_{l}
\end{aligned}
$$

Alors

$$
\frac{1}{2 \pi} \int_{0}^{2 \pi} f\left(\rho e^{i \theta}\right) e^{-i k \theta} d \theta-\left\langle\mathcal{U}_{l}, f \times \check{\mu}_{r_{1}}\right\rangle-\left\langle\mathcal{B}_{l}, f \times \check{\mu}_{r_{2}}\right\rangle=\left\langle\mathcal{T}_{l}, \check{S}_{k} \times f\right\rangle
$$

Nous allons estimer $\left\langle\mathcal{T}_{l}, \dot{S}_{k} * f\right\rangle$ en utilisant la transformation de Fourier.

On introduit unc fonction radiale $\psi_{p}$ égale à $1 \operatorname{sur} \bar{B}\left(0, \frac{1}{3} R+\frac{2}{3} R_{p}\right)$ et à support dans $\bar{B}\left(0, R_{p}^{\prime}\right)$, avec $R_{p}^{\prime}=\frac{2}{3} R+\frac{1}{3} R_{p}$, que l'on peut choisir telle que

$$
\sup _{\substack{0 \leq i, j \leq 3 \\|z| \leq R_{p}^{\prime}}}\left|\frac{\partial^{i+j}}{\partial z^{i} \partial \bar{z}^{j}} \psi_{p}(z)\right| \leq \frac{A}{(R-r)^{7}}
$$

pour une constante $A>0$ indépendante de $p$.

$$
\begin{aligned}
\left|\left\langle\mathcal{T}_{l}, \check{S}_{k} \times f\right\rangle\right| & =\left|\left\langle\mathcal{T}_{l}, \dot{S}_{k} \times\left(\psi_{p} f\right)\right\rangle\right| \\
& \leq \frac{1}{2 \pi} \int_{\mathbb{R}^{+} \times B}\left|\widetilde{\mathcal{T}}_{l}(\lambda)\left\|P_{|k|}(i \lambda)\right\| \widehat{\left(\psi_{p} f\right)}(\lambda, b)\right| \lambda \operatorname{th}\left(\frac{\pi \lambda}{2}\right) d \lambda d b \\
& \leq \frac{c^{\prime}}{2 \pi l} \int_{\mathbb{R}^{+} \times B} \|\left.\lambda\right|^{4}\left|\widehat{\left(\psi_{p} f\right)}(\lambda, b)\right| \operatorname{th}\left(\frac{\pi \lambda}{2}\right) d \lambda d b .
\end{aligned}
$$


D'autre part on $a$, pour tout $n \in \mathbb{N}$,

$$
\begin{aligned}
\mid \widehat{L^{n}\left(f \psi_{p}\right)(\lambda, b) \mid} & =\left|\left(1+\lambda^{2}\right)^{n}\right| \widehat{\left(f \psi_{p}\right)}(\lambda, b) \mid \\
& =\left|\int_{B\left(0, R_{p}^{\prime}\right)} L^{n}\left(f \psi_{p}\right)(z) e_{-i \lambda+1, b}(z) d \mu(z)\right| \\
& \leq\left(\int_{B\left(0, R_{p}^{\prime}\right)}\left|e_{-i \lambda+1, b}(z)\right| d \mu(z)\right) \sup _{z \in B\left(0, R_{p}^{\prime}\right)}\left|L^{n}\left(f \psi_{p}\right)(z)\right| \\
& \leq \pi\left(s h R_{p}^{\prime}\right)^{2} e^{R_{p}^{\prime}(1+|I m \lambda|)} \sup _{z \in B\left(0, R_{p}^{\prime}\right)}\left|L^{n}\left(f \psi_{p}\right)(z)\right|
\end{aligned}
$$

d'où, pour $n=3$, on a

$$
\begin{aligned}
\left|\left\langle\mathcal{T}_{l}, \check{S}_{k} \times f\right\rangle\right| \leq \frac{\left(s h R_{p}^{\prime}\right)^{2} c^{\prime} e^{R_{p}^{\prime}}}{2 l}\left(\int_{\mathbb{B}^{+}} \frac{\|\lambda\|^{4}}{\left(1+\lambda^{2}\right)^{3}} \operatorname{th}\left(\frac{\pi \lambda}{2}\right) d \lambda\right) \\
\sup _{z \in B\left(0, R_{p}^{\prime}\right)}\left|L^{3}\left(f \psi_{p}\right)(z)\right| \leq \frac{\gamma}{l} \frac{1}{(R-r)^{7}} \sup _{\substack{z \in B\left(0, R_{p}^{\prime}\right) \\
0 \leq i, j \leq 3}}\left|\frac{\partial^{i+j}}{\partial z^{i} \overline{\partial \bar{z}^{j}}} f(z)\right|
\end{aligned}
$$

ou $\gamma=\gamma\left(r_{1}, r_{2}, R, \varepsilon_{1}\right)>0$. Ce qui achève la preuve du théorème pour $|k| \geq 1$. Si $k=0$, on procède de la même manière en remplaçant $T_{\rho, k}$ par $\sigma_{r}$ dont la transformée sphérique est égale à $\varphi_{\lambda}^{(0,0)}(r)$.

\section{References}

1. R. ASKEY AND J. FITCH, Integral representations for Jacobi polynomials and some applications, J. Math. Anal. App. 26 (1969), 411-437.

2. C. A. BEREnStein AND R. GAY, A local version of the two circles theorem, Isr. J. Math. 55 (1986), 263-288.

3. C. A. Berenstein R. Gay and A. Yger, Invertion of the local Pompeiu transform, Journal d'Analyse Mathématique. 54 (1990), 259-287.

4. C. A. Berenstein and L. Zalcman, Pompeiu's problem on symetric spaces, Comment. Math. Helv. 55 (1980), 593-621.

5. L. Brown, B. M. Schreiber and B. A. Taylor, Spectral synthesis and Pompeiu problem, Ann. Inst. Fourier. 23 (1973), $125-154$.

6. A. ERDÉLYI (ED.), "Higher Transcendental functions," vol, 1, McGraw Hill, 1953. 
7. S. Helgason, "Groups and Geometric Analysis," Academic Press, New York, 1984.

8. G. N. Watson, Asymptotic expansions of hypergeometric functions, Trans. Combridge. Philos. Soc. 22 (1918), 277-308.

9. L. Zalcman, Analyticity and the Pompeiu problem, Arch. Rat. Mech. Anal. 47 (1972), 237-254.

UER Mathematiques

Université de Bordeaux I

351 , cours de la Liberation

33405 Talence

FRANCE

Rebut el 3 d'Agost de 1992 